\title{
COHEN-MACAULAY MODULES OVER SOME NON-REDUCED CURVE SINGULARITIES
}

\author{
IGOR BURBAN AND WASSILIJ GNEDIN
}

\begin{abstract}
In this article, we study Cohen-Macaulay modules over non-reduced curve singularities. We prove that the rings $\mathbb{k} \llbracket x, y, z \rrbracket /\left(x y, y^{q}-z^{2}\right)$ have tame Cohen-Macaulay representation type. For the singularity $\mathbb{k} \llbracket x, y, z \rrbracket /\left(x y, z^{2}\right)$ we give an explicit description of all indecomposable Cohen-Macaulay modules and apply the obtained classification to construct explicit families of indecomposable matrix factorizations of $x^{2} y^{2} \in \mathbb{k} \llbracket x, y \rrbracket$.
\end{abstract}

\section{INTRODUCTION}

Cohen-Macaulay modules over Cohen-Macaulay rings have been intensively studied in recent years. They appear in the literature in various incarnations like matrix factorizations, objects of the triangulated category of singularities or lattices over orders.

Our interest to Cohen-Macaulay modules is representation theoretic. In the case of a reduced curve singularity, the behavior of the representation type of the category of Cohen-Macaulay modules $\mathrm{CM}(A)$ is completely understood. Assume, for simplicity, that $A$ is an algebra over an algebraically closed field $\mathbb{k}$ of characteristic zero.

- According to Drozd and Roiter [17, Jacobinski [21] and Greuel and Knörrer [19], $\mathrm{CM}(A)$ is representation finite if and only if $A$ dominates a simple curve singularity. See also the expositions in the monographs [25] and [27].

- Drozd and Greuel have proven in [15] that $\operatorname{CM}(A)$ is tame if and only if $A$ dominates a singularity of type

$$
\mathrm{T}_{p q}(\lambda)=\mathbb{k} \llbracket x, y \rrbracket /\left(x^{p-2}-y^{2}\right)\left(x^{2}-\lambda y^{q-2}\right) \quad \begin{cases}\frac{1}{p}+\frac{1}{q}=\frac{1}{2}, & \lambda \in \mathbb{k} \backslash\{0,1\}, \\ \frac{1}{p}+\frac{1}{q}<\frac{1}{2}, & \lambda=1 .\end{cases}
$$

In particular, they have shown that the singularities

$$
\mathrm{P}_{p q}=\mathbb{k} \llbracket x, y, z \rrbracket /\left(x y, x^{p}+y^{q}-z^{2}\right), \quad \text { where } p, q \in \mathbb{N}_{\geq 2},
$$

are Cohen-Macaulay tame.

- A reduced curve singularity which neither dominates a simple nor a $\mathrm{T}_{p q}(\lambda)$ singularity has wild Cohen-Macaulay representation type [14].

- There are also other approaches to establish tameness of $\operatorname{CM}\left(\mathrm{T}_{p q}(\lambda)\right)$ : one using the generalized geometric McKay Correspondence [22, 16] and another via clustertilting theory [12].

The following results about the representation type of a non-reduced curve singularity are known so far.

- By a theorem of Auslander [2, a non-reduced curve singularity always has infinite Cohen-Macaulay representation type.

- Buchweitz, Greuel and Schreyer have shown in [9] that the singularities $\mathrm{A}_{\infty}=$ $\mathbb{k} \llbracket x, y \rrbracket /\left(y^{2}\right)$ and $\mathrm{D}_{\infty}=\mathbb{k} \llbracket x, y \rrbracket /\left(x y^{2}\right)$ have discrete Cohen-Macaulay representation type. 
- Leuschke and Wiegand have proven in [26] that $\mathrm{A}_{\infty}, \mathrm{D}_{\infty}$ and $\mathbb{k} \llbracket x, y, z \rrbracket /\left(x y, y z, z^{2}\right)$ are the only curve singularities of bounded but infinite Cohen-Macaulay type.

- Burban and Drozd have proven in [11] that the hypersurface singularities $\mathrm{T}_{\infty q}=$ $\mathbb{k} \llbracket x, y \rrbracket /\left(x^{2} y^{2}-y^{q}\right)$, where $q \in \mathbb{N}_{\geq 3}$, (respectively $\mathrm{T}_{\infty \infty}=\mathbb{k} \llbracket x, y \rrbracket /\left(x^{2} y^{2}\right)$ ) are Cohen-Macaulay tame (under the additional assumption that $\operatorname{char}(\mathbb{k})=0$, respectively $\operatorname{char}(\mathbb{k}) \neq 2$ ). However, an explicit description of the corresponding indecomposable matrix factorizations is still not known.

In this article, we obtain the following results.

1. First, we prove (see Theorem 2.1) that the curve singularities

$$
\mathrm{P}_{\infty q}=\mathbb{k} \llbracket x, y, z \rrbracket /\left(x y, y^{q}-z^{2}\right) \quad \text { and } \quad \mathrm{P}_{\infty \infty}=\mathbb{k} \llbracket x, y, z \rrbracket /\left(x y, z^{2}\right)
$$

are Cohen-Macaulay tame for any algebraically closed field $\mathbb{k}$ of any characteristic (in the case $\operatorname{char}(\mathbb{k})=2$ the definition of $\mathrm{P}_{\infty q}$ has to be modified, see Remark 2.5). The method of the proof extends the approach of Drozd and Greuel [15] to the case of non-reduced curve singularities and is based on Bondarenko's work on representations of bunches of semi-chains [6]. Our approach can be summarized by the following diagram of categories and functors:

$$
\mathrm{CM}(\mathrm{P}) \stackrel{\mathbb{I}}{\longleftarrow} \mathrm{CM}(\mathrm{R}) \underset{\mathbb{G}}{\stackrel{\mathbb{F}}{\sim}} \operatorname{Tri}(\mathrm{R}) \stackrel{\mathbb{P}}{\longrightarrow} \operatorname{Rep}(\mathfrak{B}) .
$$

We start with a singularity $\mathrm{P}=\mathrm{P}_{\infty q}$ or $\mathrm{P}_{\infty \infty}$ and replace it by its minimal overring $\mathrm{R}$. The forgetful functor $\mathbb{I}$ embeds $C M(R)$ into $C M(P)$ as a full subcategory. By a result of Bass [3], the "difference" between $\mathrm{CM}(\mathrm{R})$ and $\mathrm{CM}(\mathrm{P})$ is very small. The category of triples Tri $(R)$ plays a key role in our approach. According to [11], the functors $\mathbb{F}$ and $\mathbb{G}$ are quasi-inverse equivalences of categories. Finally, $\operatorname{Rep}(\mathfrak{B})$ is a certain bimodule category in the sense of [13]. The functor $\mathbb{P}$ preserves isomorphy classes and indecomposability of objects. We prove that $\operatorname{Rep}(\mathfrak{B})$ is the category of representations of a certain bunch of semi-chains. According to a theorem of Bondarenko [6], $\operatorname{Rep}(\mathfrak{B})$ is representation tame. This implies tameness of $\mathrm{CM}(\mathrm{P})$.

2. Next, we show how to pass from canonical forms describing indecomposable objects of $\operatorname{Rep}(\mathfrak{B})$ to a concrete description of the corresponding indecomposable Cohen-Macaulay $\mathrm{P}$-modules. We illustrate this technique giving an explicit description of the indecomposable Cohen-Macaulay modules over $\mathrm{P}_{\infty \infty}$. They are described in terms of quite transparent combinatorial data: bands and strings, see Theorem 3.19. The obtained classification turns out to be perfectly adapted to separate those Cohen-Macaulay modules which are locally free on the punctured spectrum from those which are not, see Remark 3.17 .

3. At last, we construct explicit families of indecomposable matrix factorizations of $x^{2} y^{2} \in$ $\mathbb{k} \llbracket x, y \rrbracket$. In this context, there is the following diagram of categories and functors:

$$
\mathrm{CM}(\mathrm{R}) \stackrel{\mathbb{J}}{\longrightarrow} \mathrm{CM}(\mathrm{T}) \longrightarrow \underline{\mathrm{MF}}\left(x^{2} y^{2}\right) .
$$

Here, $\mathrm{R}$ is the minimal overring of $\mathrm{P}_{\infty \infty}$, the functor $\mathbb{J}$ is a fully faithful embedding, $\mathrm{T}=\mathbb{k} \llbracket x, y \rrbracket /\left(x^{2} y^{2}\right)$ and $\mathrm{MF}\left(x^{2} y^{2}\right)$ is the homotopy category of matrix factorizations of $x^{2} y^{2}$ (which is equivalent to the stable category $\mathrm{CM}(\mathrm{T})$ by a result of Eisenbud [18]). Results of this article provide a partial classification of the indecomposable objects of $\underline{\operatorname{MF}}\left(x^{2} y^{2}\right)$ as well as an equivalent category $\underline{\operatorname{MF}}\left(x^{2} y^{2}+u v\right)$. 
Acknowledgement. This research was supported by the DFG project $\mathrm{Bu}-1866 / 2-1$. We are also thankful to Lesya Bodnarchuk for supplying us with TikZ pictures illustrating the technique of matrix problems.

\section{Survey on Cohen-Macaulay modules over Curve Singularities}

In this section we collect definitions and some known facts on Cohen-Macaulay modules over curve singularities. The proofs of the mentioned statements can be found in the monographs [7, 25, 27], see also the survey article [10].

1.1. Definitions and basic properties. Let $(A, \mathfrak{m})$ be a local Noetherian ring of Krull dimension one (a curve singularity), $\mathbb{k}=A / \mathfrak{m}$ its residue field and $Q=Q(A)$ its total ring of fractions.

Definition 1.1. A curve singularity $A$ is

- Cohen-Macaulay if and only if $\operatorname{Hom}_{A}(\mathbb{k}, A)=0$ (equivalently, $A$ contains a regular element).

- Gorenstein if and only if it is Cohen-Macaulay and $\operatorname{Ext}_{A}^{1}(\mathbb{k}, A) \cong \mathbb{k}_{\mathrm{k}}$ (equivalently, $\operatorname{inj.} \operatorname{dim}_{A}(A)=1$ ).

Note that a reduced curve singularity is automatically Cohen-Macaulay. However, in this article we mainly focus on non-reduced ones.

Lemma 1.2. Let $A$ be a Cohen-Macaulay curve singularity. Then $Q$ is an Artinian ring. Moreover, if $\left\{\mathfrak{p}_{1}, \ldots, \mathfrak{p}_{t}\right\}$ is the set of minimal prime ideals of $A$ then there exists a ring isomorphism $\gamma: Q \longrightarrow A_{\mathfrak{p}_{1}} \times \cdots \times A_{\mathfrak{p}_{t}}$ making the following diagram

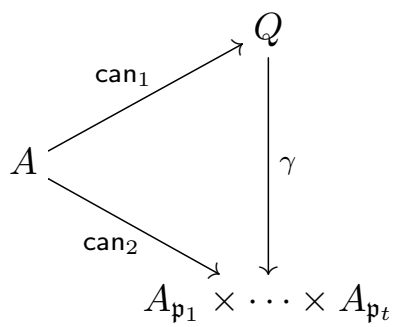

commutative, where $\operatorname{can}_{1}$ and $\mathrm{can}_{2}$ are canonical morphisms.

Proof. Since $A$ is Cohen-Macaulay, the associator of $A$ coincides with $\left\{\mathfrak{p}_{1}, \ldots, \mathfrak{p}_{n}\right\}$. By [4, Chapter IV, Proposition 2.5.10] $Q$ is Artinian and its maximal ideals are $\mathfrak{p}_{1} Q, \ldots, \mathfrak{p}_{n} Q$. Hence, $Q \cong Q_{\mathfrak{p}_{1} Q} \times \cdots \times Q_{\mathfrak{p}_{n} Q}$. Since $Q_{\mathfrak{p}_{i} Q}=A_{\mathfrak{p}_{i}}$ for $1 \leq i \leq n$, the result follows.

Definition 1.3. For an $A$-module $M$ we set

$$
\Gamma_{\mathfrak{m}}(M):=\left\{x \in M \mid \mathfrak{m}^{t} x=0 \text { for some } t \in \mathbb{N}\right\} .
$$

The following result can be easily deduced from Lemma 1.2

Lemma 1.4. Let $A$ be a Cohen-Macaulay curve singularity. For a Noetherian A-module $M$ we have:

$$
\Gamma_{\mathfrak{m}}(M)=\operatorname{ker}\left(M \longrightarrow Q \otimes_{A} M\right)=: \operatorname{tor}(M) .
$$

Moreover, the following statements are equivalent:

- $\operatorname{Hom}_{A}(\mathbb{k}, M)=0$.

- $M$ is torsion free, i.e. $\operatorname{tor}(M)=0$. 
Definition 1.5. A Noetherian module $M$ satisfying the conditions of Lemma 1.4 is called maximal Cohen-Macaulay. In what follows we just say that $M$ is Cohen-Macaulay. In this case, the $Q$-module $Q(M)$ is called the rational envelope of $M$. More generally, a Noetherian module $N$ over a Noetherian ring $S$ (say, of Krull dimension one) is (maximal) Cohen-Macaulay if for any maximal ideal $\mathfrak{n}$ in $S$ the localization $N_{\mathfrak{n}}$ is Cohen-Macaulay. In what follows, $\operatorname{CM}(S)$ denotes the category of Cohen-Macaulay $S$-modules.

Lemma 1.6. Assume that a Cohen-Macaulay curve singularity $A$ is Gorenstein in codimension zero (i.e. $Q$ is self-injective). Then for a Noetherian $A$-module $M$, the following conditions are equivalent:

- $M$ is Cohen-Macaulay.

- $M$ embeds into a finitely generated free A-module.

A proof of this Lemma can be found in [25, Appendix A, Corollary 15].

Remark 1.7. The statement of Lemma 1.6 is not true for an arbitrary Cohen-Macaulay curve singularity. For example, let $A=\mathbb{k} \llbracket x, y, z \rrbracket /\left(x^{2}, x y, y^{2}\right)$ and $K$ be a canonical $A$ module. Then $K$ does not embed into a free $A$-module.

Definition 1.8. A ring $R$ is an overring of $A$ if $A \subseteq R \subset Q$ and the ring extension $A \subseteq R$ is finite. We also say that $R$ birationally dominates $A$.

Proposition 1.9. Let $A$ be a Cohen-Macaulay curve singularity and $R$ an overring of $A$. Then the following results are true.

- $R$ is Cohen-Macaulay.

- We have an adjoint pair $\left(R \bigotimes_{A}-, \mathbb{I}(-)\right)$, where $\mathbb{I}: \mathrm{CM}(R) \longrightarrow \mathrm{CM}(A)$ is the restriction (or forgetful) functor and $R \otimes_{A}-: \mathrm{CM}(A) \longrightarrow \mathrm{CM}(R)$ sends a CohenMacaulay module $M$ to $R \otimes_{A} M / \operatorname{tor}\left(R \otimes_{A} M\right)$.

- II is fully faithful.

- If $M=\left\langle w_{1}, \ldots, w_{t}\right\rangle_{A} \subset Q^{n}$, then $R \otimes_{A} M \cong R \cdot M:=\left\langle w_{1}, \ldots, w_{t}\right\rangle_{R} \subset Q^{n}$.

Proof. The first statement follows from the fact that $\operatorname{depth}_{A}(R)=1=\operatorname{depth}_{R}(R)$. The second result follows from the functorial isomorphisms

$$
\operatorname{Hom}_{R}\left(R \otimes_{A} M, N\right) \cong \operatorname{Hom}_{R}\left(R \otimes_{A} M, N\right) \cong \operatorname{Hom}_{A}(M, \mathbb{I}(N)) .
$$

For a proof of the third statement, see for example [25, Lemma 4.14]. The fourth result follows from the fact that the kernel of the natural morphism $R \otimes_{A} M \longrightarrow R \cdot M$ is $\operatorname{tor}\left(R \otimes_{A} M\right)$.

Corollary 1.10. Let $A$ be a Cohen-Macaulay curve singularity and $R$ be an overring of A. Then the following statements are true.

- Let $N_{1}$ and $N_{2}$ be Cohen-Macaulay $R$-modules. Then $N_{1} \cong N_{2}$ if and only if $\mathbb{I}\left(N_{1}\right) \cong \mathbb{I}\left(N_{2}\right)$ in $\operatorname{CM}(A)$.

- A Cohen-Macaulay $R$-module $N$ is indecomposable if and only if $N$ is indecomposable viewed as an $A$-module.

The following result is due to Bass [3, Proposition 7.2], see also [25, Lemma 4.9].

Theorem 1.11. Let $(A, \mathfrak{m})$ be a Gorenstein curve singularity and let $R=\operatorname{End}_{A}(\mathfrak{m})$. Then the following results are true.

- $R \cong\{r \in Q \mid r \mathfrak{m} \subseteq \mathfrak{m}\}$. In particular, $R$ is an overring of $A$. 
- If $A$ is not regular, we have an exact sequence of $A$-modules

$$
0 \longrightarrow A \stackrel{\imath}{\longrightarrow} R \longrightarrow \mathbb{k} \longrightarrow 0
$$

where $\imath$ is the canonical inclusion. This short exact sequence defines a generator of the $A$-module $\operatorname{Ext}_{A}^{1}(\mathbb{k}, A) \cong \mathbb{k}$.

- In the latter case, let $S$ be any other proper overring of $A$. Then $S$ contains $R$. In other words, $R$ is the minimal overring of the curve singularity $A$.

- Let $M$ be a Cohen-Macaulay A-module without free direct summands. Then there exists a Cohen-Macaulay $R$-module $N$ such that $M=\mathbb{I}(N)$.

Remark 1.12. Theorem 1.11 gives a precise measure of the representation theoretic difference between the categories $\mathrm{CM}(A)$ and $\mathrm{CM}(R)$. Namely, an indecomposable CohenMacaulay $A$-module $M$ is either regular or is the restriction of an indecomposable CohenMacaulay $R$-module. In more concrete terms, assume that $M=\left\langle w_{1}, \ldots, w_{t}\right\rangle_{A} \subset Q^{n}$ contains no free direct summands (according to Lemma 1.6, any Cohen-Macaulay $A-$ module admits such embedding). Then $M=\left\langle w_{1}, \ldots, w_{t}\right\rangle_{R}$.

Proposition 1.13. In the situation of Theorem 1.11, assume that $N=\left\langle w_{1}, \ldots, w_{t}\right\rangle_{R} \subset$ $Q^{n}$ is an indecomposable Cohen-Macaulay $R$-module. Then either $N \cong R$ or $N=$ $\left\langle w_{1}, \ldots, w_{t}\right\rangle_{A}$.

Proof. Pose $M:=\left\langle w_{1}, \ldots, w_{t}\right\rangle_{A}$. Obviously, we have: $N=R \cdot M$. If $M$ contains a free direct summand, i.e. $M \cong M^{\prime} \oplus A^{m}$ then $N=R \cdot M \cong R \cdot M^{\prime} \oplus R^{m}$. As $N$ is assumed to be indecomposable, $N \cong R$. If $N \neq R$, then $M$ has no free direct summands. Hence, by Theorem 1.11 and Remark 1.12 we have: $R \cdot M=M$.

Definition 1.14. A Cohen-Macaulay $A$-module $M$ is locally free on the punctured spectrum of $A$ if for any minimal prime ideal $\mathfrak{p}$ in $A$ the localization $M_{\mathfrak{p}}$ is free over $A_{\mathfrak{p}}$.

Remark 1.15. According to Lemma 1.2, a Cohen-Macaulay $A$-module $M$ is locally free on the punctured spectrum if and only if its rational envelope $Q(M)$ is projective over $Q$.

In what follows, $\mathrm{CM}^{\text {lf }}(A)$ denotes the category of Cohen-Macaulay $A$-modules which are locally free on the punctured spectrum.

1.2. Category of triples. Let $(R, \mathfrak{m})$ be a Cohen-Macaulay curve singularity, $S$ an overring of $R$ and $I=\operatorname{ann}_{R}(S / R)$ the corresponding conductor ideal. The next result is straightforward, see for example [11, Lemma 12.1].

Lemma 1.16. The following statements are true.

- $I=I R=I S$. In other words, $I$ is an ideal both in $R$ and in $S$. Moreover, $I$ is the biggest ideal having this property.

- The rings $\bar{R}=R / I$ and $\bar{S}=S / I$ are Artinian.

For a Cohen-Macaulay $A$-module $M$ we denote

- $\tilde{M}:=S \otimes_{R} M \in \mathrm{CM}(S)$.

- $\bar{M}:=\bar{R} \otimes_{R} M \in \bmod (\bar{R})$.

- $\check{M}:=\bar{S} \otimes_{S} \tilde{M} \in \bmod (\bar{S})$.

Then the following result is true, see [11, Lemma 12.2].

Lemma 1.17. The canonical map $\theta_{M}: \bar{S} \otimes_{\bar{R}} \bar{M} \longrightarrow \check{M}$ is surjective and its adjoint map $\tilde{\theta}_{M}: \bar{M} \longrightarrow \check{M}$ is injective. 
Definition 1.18. Consider the following category of triples Tri $(R)$. Its objects are triples $(N, V, \theta)$, where

- $N$ is a maximal Cohen-Macaulay $S$-module,

- $V$ is a Noetherian $\bar{R}$-module,

- $\theta: \bar{S} \otimes_{\bar{R}} V \rightarrow \bar{S} \otimes_{S} N$ is an epimorphism of $\bar{S}$-modules such that the adjoint morphism of $\bar{R}$-modules $\tilde{\theta}: V \rightarrow \bar{S} \otimes_{\bar{R}} V \stackrel{\theta}{\longrightarrow} \bar{S} \otimes_{S} N$ is a monomorphism.

A morphism between two triples $(N, V, \theta)$ and $\left(N^{\prime}, V^{\prime}, \theta^{\prime}\right)$ is given by a pair $(\psi, \varphi)$, where

- $\psi: N \rightarrow N^{\prime}$ is a morphism of $S$-modules and

- $\varphi: V \rightarrow V^{\prime}$ is a morphism of $\bar{R}$-modules

such that the following diagram of $\bar{S}$-modules is commutative:

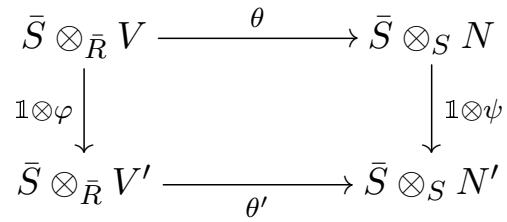

Definition 1.18 is motivated by the following theorem, see [11, Theorem 12.5].

Theorem 1.19. The functor $\mathbb{F}: \mathrm{CM}(R) \longrightarrow \operatorname{Tri}(R)$ mapping a maximal Cohen-Macaulay module $M$ to the triple $\left(\tilde{M}, \bar{M}, \theta_{M}\right)$, is well-defined and is an equivalence of categories. A quasi-inverse functor $\mathbb{G}: \operatorname{Tri}(R) \longrightarrow \mathrm{CM}(R)$ is defined as follows. Let $\mathrm{T}=(N, V, \theta)$ be an object of $\operatorname{Tri}(R)$. Then $M^{\prime}=\mathbb{G}(\mathrm{T}):=\pi^{-1}(\operatorname{Im}(\tilde{\theta})) \subseteq N$, where $\pi: N \rightarrow \bar{N}:=N / I N$ is the canonical projection. In other words, we have the following commutative diagram

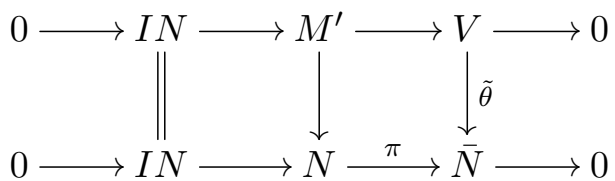

in the category of $R$-modules.

In many cases, Theorem 1.19 provides an efficient tool to reduce the classification of indecomposable objects of $\mathrm{CM}(R)$ to a certain problem of linear algebra (a matrix problem).

Remark 1.20. There are several variations of the construction appearing in Theorem 1.19, see [11, Appendix A] for an account of them.

1.3. Cohen-Macaulay modules over simple curve singularities of type A. Let $\mathbb{k}$ be an algebraically closed field. For simplicity, let us additionally assume that $\operatorname{char}(\mathbb{k}) \neq 2$, see however Remark 1.22 . For any $m \in \mathbb{N}$, denote

$$
S=\mathrm{A}_{m}:=\mathbb{k} \llbracket x, u \rrbracket /\left(x^{m+1}-u^{2}\right)
$$

the corresponding simple curve singularity of type $A_{m}$. The following is essentially due to Bass [3], see also [25, 27].

Theorem 1.21. The indecomposable Cohen-Macaulay $S$-modules have the following description.

- Assume $m=2 n, n \in \mathbb{N}$. For any $1 \leq i \leq n$ consider the ideal $X_{i}:=\left(x^{i}, u\right)$. Then $X_{0}=(1)=S, X_{1}, \ldots, X_{n}$ is the complete list of indecomposable objects of $\mathrm{CM}(S)$. 
Moreover, the Auslander-Reiten quiver of $\operatorname{CM}(S)$ has the form

$$
\left.X_{0} \stackrel{\cdot x}{\rightleftarrows \iota} X_{1} \stackrel{\cdot x}{\rightleftarrows \iota} X_{2} \underset{\iota}{\stackrel{\cdot x}{\rightleftarrows}} \ldots \stackrel{\cdot x}{\longleftarrow \iota} X_{n}\right) \pi
$$

Here, $\imath$ denotes the inclusion of ideals and $x$. is the multiplication by $x$. The endomorphism $\pi \in \operatorname{End}_{S}\left(X_{n}\right)$ is defined as follows: $\pi\left(x^{n}\right)=u$ and $\pi(u)=x^{n+1}$.

- Assume $m=2 n+1, n \in \mathbb{N}_{0}$. Again, for any $1 \leq i \leq n$ consider $X_{i}:=\left(x^{i}, u\right) \subset$ $S$. Additionally, denote $X_{n+1}^{ \pm}:=\left(x^{n+1} \pm u\right)$. Then the indecomposable CohenMacaulay $S$-modules are $X_{0}=(1)=S, X_{1}, \ldots, X_{n}, X_{n+1}^{+}$and $X_{n+1}^{-}$. Moreover, the Auslander-Reiten quiver of $\mathrm{CM}(S)$ is in this case

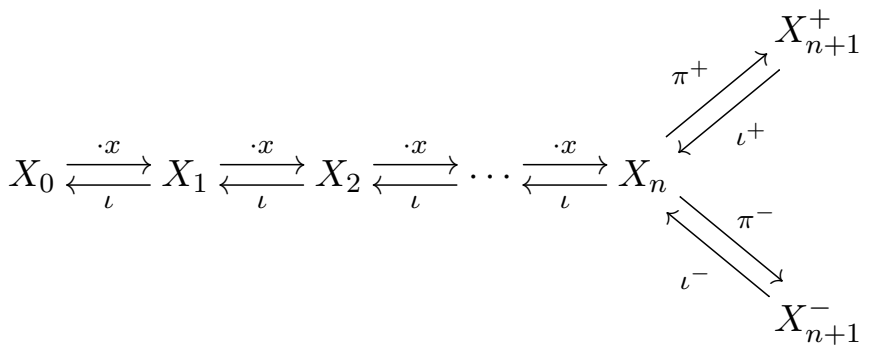

Here, $\iota$ and $\iota^{ \pm}$denote inclusions of ideals, $x$. stands for multiplication by $x$. The maps $\pi^{ \pm}: X_{n} \longrightarrow X_{n+1}^{ \pm}$are defined as follows: $\pi^{ \pm}\left(x^{n}\right)=\left(x^{n+1} \pm u\right)$ and $\pi^{ \pm}(u)=$ $x\left(x^{n+1} \pm u\right)$.

Remark 1.22. In the case $\operatorname{char}(\mathbb{k})=2$ there are the following subtleties in defining simple curve singularities of type $\mathrm{A}_{m}$.

- For $m=2 n+1$ one should take the ring $\mathrm{A}_{2 n+1}=\mathbb{k} \llbracket x, u \rrbracket /\left(u\left(u-x^{n+1}\right)\right)$ (the ring defined by (1.2) is no longer reduced!). In this case, one should pose $X_{n+1}^{+}:=(u)$ and $X_{n+1}^{-}:=\left(u-x^{n+1}\right)$. Then the indecomposable Cohen-Macaulay modules are $X_{0}, \ldots, X_{n}, X_{n+1}^{ \pm}$, where $X_{i}$ has the same definition as in the case $\operatorname{char}(\mathbb{k}) \neq 2$ for $0 \leq i \leq n$.

- For $m=2 n$ there are more simple singularities than in the case $\operatorname{char}(\mathbb{k}) \neq 2$. Namely, for $1 \leq s \leq n-1$ consider the ring $\mathrm{A}_{2 n}^{s}=\mathbb{k} \llbracket x, u \rrbracket /\left(u^{2}+x^{2 n+1}+u x^{n+s}\right)$. Then $\mathrm{A}_{2 n}^{s} ¥ \mathrm{~A}_{2 n}^{t}$ for any $1 \leq s \neq t \leq n-1$. Moreover, $\mathrm{A}_{2 n}^{s} \neq \mathrm{A}_{2 n}$ for any $1 \leq s \leq n-1$. However, the description of indecomposable Cohen-Macaulay modules over $\mathrm{A}_{2 n}^{s}$ is essentially the same as over $\mathrm{A}_{2 n}$, see [3] and [23]. In particular, the Auslander-Reiten quivers of $\mathrm{A}_{2 n}^{s}$ and $\mathrm{A}_{2 n}$ coincide.

The following result is due to Buchweitz, Greuel and Schreyer [9, Section 4.1].

Theorem 1.23. For an algebraically closed field $\mathbb{k}$ (of arbitrary characteristic) let $S=$ $\mathrm{A}_{\infty}:=\mathbb{k} \llbracket x, u \rrbracket /\left(u^{2}\right)$. Then the indecomposable Cohen-Macaulay $S$-modules are $X_{0}, X_{1}$, $\ldots, X_{\infty}$, where $X_{0}=(1)=S, X_{\infty}=(u)$ and $X_{i}=\left(x^{i}, u\right)$ for $i \in \mathbb{N}$. In particular, $X_{\infty}$ is the only indecomposable Cohen-Macaulay $S$-module which is not locally free on the punctured spectrum of $S$. The Auslander-Reiten quiver of the category $\operatorname{CM}^{\text {lf }}(S)$ has the form

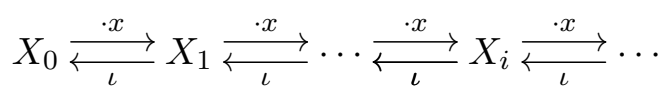

Remark 1.24. It is natural to extend the quiver (1.5) with the remaining indecomposable Cohen-Macaulay $S$-module $X_{\infty}$. Moreover, for any $i \in \mathbb{N}_{0}$ denote $\pi_{i}: X_{i} \longrightarrow X_{\infty}$ the 
map sending $x^{i}$ to $u$ and $u$ to 0 . Of course, $\pi_{i+1}=x \cdot \pi_{i}$ for any $i \in \mathbb{N}_{0}$. The entire structure of the category $\mathrm{CM}(S)$ can be visualized by the diagram:

$$
X_{0} \stackrel{\cdot x}{\longleftarrow \iota} X_{1} \underset{\iota}{\stackrel{\cdot x}{\rightleftarrows}} \cdots \underset{\iota}{\stackrel{\cdot x}{\longleftarrow}} X_{i} \stackrel{\cdot x}{\longleftarrow \iota} \ldots \frac{\pi}{\longleftarrow} \ldots X_{\infty}
$$

Definition 1.25. Let $(A, \mathfrak{m})$ be a Cohen-Macaulay curve singularity. Consider the category $\overline{\mathrm{CM}}(A)$ defined as follows:

- $\mathrm{Ob}(\overline{\mathrm{CM}}(A))=\mathrm{Ob}(\mathrm{CM}(A))$.

- For $M, N \in \mathrm{Ob}(\overline{\mathrm{CM}}(A))$ we set

$$
\overline{\operatorname{Hom}}_{A}(M, N)=\operatorname{Im}\left(\operatorname{Hom}_{A}(M, N) \longrightarrow \operatorname{Hom}_{\mathbb{k}}\left(M \otimes_{A} \mathbb{k}, N \otimes_{A} \mathbb{k}\right)\right) .
$$

- The composition of morphisms in $\overline{\mathrm{CM}}(A)$ is induced by the composition of morphisms in $\mathrm{CM}(A)$.

The following result is straightforward.

Lemma 1.26. The canonical projection functor $\mathrm{CM}(A) \rightarrow \overline{\mathrm{CM}}(A)$ is full and respects isomorphy classes of objects. Moreover, if $S$ is a curve singularity of type $\mathrm{A}_{m}$ for some $m \in \mathbb{N}^{*} \cup\{\infty\}$, then $\overline{\mathrm{CM}}(S)$ is equivalent to the additive closure of the path algebra category of the corresponding Auslander-Reiten quivers (1.3), (1.4) respectively (1.6) subject to the following zero relations:

- $(\cdot x) \circ \imath=\imath \circ(\cdot x)=0$.

- The inclusion $\imath: X_{1} \rightarrow X_{0}$ is zero in $\overline{\mathrm{CM}}(S)$.

• $\begin{cases}\pi^{2}=0, & \text { if } m \text { is even, } \\ \pi^{ \pm} \circ \imath^{+}=\pi^{ \pm} \circ \imath^{-}=\imath^{+} \circ \pi^{+}+\imath^{-} \circ \pi^{-}=0, & \text { if } m \text { is odd, } \\ \pi \circ \iota=0, & \text { if } m=\infty .\end{cases}$

\section{TAMeness of $\mathrm{CM}\left(\mathrm{P}_{\infty q}\right)$}

Let $\mathbb{k}$ be an algebraically closed field such that $\operatorname{char}(\mathbb{k}) \neq 2$ and $p, q \in \mathbb{N}_{\geq 2}$. Consider the curve singularity

$$
\mathrm{P}_{p q}:=\mathbb{k} \llbracket x, y, z \rrbracket /\left(x y, x^{p}+y^{q}-z^{2}\right) .
$$

By a result of Drozd and Greuel [15, Section 3], the category $\mathrm{CM}\left(\mathrm{P}_{p q}\right)$ is representation tame. For any $q \in \mathbb{N}_{\geq 2}$ consider the limiting non-reduced singularity

$$
\mathrm{P}_{\infty q}:=\mathbb{k} \llbracket x, y, z \rrbracket /\left(x y, y^{q}-z^{2}\right)
$$

as well as the "largest degeneration" $\mathrm{P}_{\infty \infty}:=\mathbb{k} \llbracket x, y, z \rrbracket /\left(x y, z^{2}\right)$ of the family (2.1).

The first major result of this article is the following.

Theorem 2.1. The non-reduced curve singularities $\mathrm{P}_{\infty q}$ have tame Cohen-Macaulay representation type for any $q \in \mathbb{N}_{\geq 2} \cup\{\infty\}$.

Proof. 1. Since $\mathrm{P}:=\mathrm{P}_{\infty q}$ is a complete intersection, it is Gorenstein. Let $\mathrm{R}=\operatorname{End}_{\mathrm{P}}(\mathfrak{m})$ be the minimal overring of $\mathrm{P}$. The first statement of Theorem 1.11 implies that

$$
\mathrm{R} \cong \mathbb{k} \llbracket x, y, u, v \rrbracket /\left(x y, y u, u v, v x, u^{2}, y^{q}-v^{2}\right),
$$

where the canonical inclusion $\imath: \mathrm{P} \longrightarrow \mathrm{R}$ maps $x$ to $x, y$ to $y$ and $z$ to $u+v$ (in what follows, the generator $y^{q}-v^{2}$ has to be replaced by $v^{2}$ for $\left.q=\infty\right)$. Note that $u=\frac{x z}{x+y}$ 
and $v=\frac{y z}{x+y}$ if we regard $\mathrm{R}$ as a subring of the total ring of fractions $\mathrm{Q}=\mathrm{Q}(\mathrm{P})$. According to Theorem 1.11, any non-regular indecomposable Cohen-Macaulay $\mathrm{P}$-module is a restriction of some indecomposable Cohen-Macaulay $\mathrm{R}$-module. Thus, it has to be shown that the category $\mathrm{CM}(\mathrm{R})$ has tame representation type.

2. Next, note that $\mathbf{S}=\mathbb{k} \llbracket x, u \rrbracket /\left(u^{2}\right) \times \mathbb{k} \llbracket y, v \rrbracket /\left(y^{q}-v^{2}\right)$ is an overring of $\mathrm{R}$. Indeed, we have an inclusion $S \subset \mathrm{Q}$, where both idempotents of $\mathrm{R}$ can be expressed as follows:

$$
e_{x}:=(1,0)=\frac{x}{x+y} \quad \text { and } \quad e_{y}:=(0,1)=\frac{y}{x+y} .
$$

The reason to pass from $\mathrm{P}$ to its overring $\mathrm{R}$ is explained by the following observation: the conductor ideal $I:=\operatorname{ann}_{\mathrm{R}}(\mathrm{S} / \mathrm{R})$ coincides with the maximal ideal $\mathfrak{m}=(x, y, u, v)_{\mathrm{R}}$. Hence, $\overline{\mathrm{R}}:=\mathrm{R} / I \cong \mathbb{k}$ and $\overline{\mathrm{S}}:=\mathrm{S} / I \cong \mathbb{k}_{x} \times \mathbb{k}_{y}=\mathbb{k} \times \mathbb{k}$. Under this identification, the canonical inclusion $\overline{\mathrm{R}} \rightarrow \overline{\mathrm{S}}$ is identified with the diagonal embedding.

According to Theorem 1.19 , the category $\mathrm{CM}(\mathrm{R})$ is equivalent to the category of triples $\operatorname{Tri}(\mathrm{R})$. Thus, we have to show tameness of $\operatorname{Tri}(\mathrm{R})$. Let $\mathrm{T}=(N, V, \theta)$ be a triple of $\mathrm{R}$. Then the following facts are true.

- Since $V$ is just a module over $\overline{\mathrm{R}} \cong \mathbb{k}$, we have: $V \cong \mathbb{k}^{t}$ for some $t \in \mathbb{N}_{0}$.

- Denote $\mathrm{S}_{x}=\mathbb{k} \llbracket x, u \rrbracket /\left(u^{2}\right)$ and $\mathbf{S}_{y}=\mathbb{k} \llbracket y, v \rrbracket /\left(y^{q}-v^{2}\right)$. Then $\mathbf{S}=\mathbf{S}_{x} \times \mathbf{S}_{y}$ and $N \cong N_{x} \oplus N_{y}$, where $N_{x} \in \mathrm{CM}\left(\mathrm{S}_{x}\right)$ and $N_{y} \in \mathrm{CM}\left(\mathrm{S}_{y}\right)$. According to Theorem 1.21 and Theorem 1.23, the Cohen-Macaulay modules $N_{x}$ and $N_{y}$ split into a direct sum of ideals

$-X_{0}=\left(e_{x}\right)_{\mathrm{S}} \cong \mathrm{S}_{x}, X_{i}=\left(x^{i}, u\right)_{\mathrm{S}}$, for $i \in \mathbb{N}^{*}$ and $X_{\infty}=(u)$.

$-Y_{0}=\left(e_{y}\right)_{\mathrm{S}} \cong \mathrm{S}_{y}, Y_{j}=\left(y^{j}, v\right)$ for $1 \leq j \leq s-1$ and $Y_{s}^{ \pm}=\left(y^{s} \pm v\right)_{\mathrm{S}}$ if $q=2 s$ is even, respectively $Y_{s}=\left(y^{s}, v\right)$ if $q=2 s+1$ is odd.

- We have: $\bar{N}_{x}=N_{x} / I N_{x} \cong \mathbb{k}_{x}^{m}$ and $\bar{N}_{y}=N_{y} / I N_{y} \cong \mathbb{k}_{y}^{n}$ for some $m, n \in \mathbb{N}_{0}$. In what follows, we choose bases of $\bar{N}_{x}$ and $\bar{N}_{y}$ induced by the distinguished generators of the ideals which occur in a direct sum decomposition of $N_{x}$ and $N_{y}$. Thus, the gluing map $\theta: \overline{\mathrm{S}} \otimes_{\overline{\mathrm{R}}} V \longrightarrow N / I N$ is given by a pair of matrices $\left(\Theta_{x}, \Theta_{y}\right) \in \operatorname{Mat}_{m \times t}(\mathbb{k}) \times \operatorname{Mat}_{n \times t}(\mathbb{k})$.

- The condition that the morphism of $\overline{\mathrm{S}}$-modules $\theta$ is surjective just means that both matrices $\Theta_{x}$ and $\Theta_{y}$ have full row rank. The condition that $\tilde{\theta}$ is injective is equivalent to say that the matrix $\tilde{\Theta}:=\left(\begin{array}{c}\Theta_{x} \\ \Theta_{y}\end{array}\right)$ has full column rank.

3. Let us now describe the matrix problem underlying a description of isomorphy classes of objects of Tri(R). If two triples $\mathrm{T}=(N, V, \theta)$ and $\mathrm{T}^{\prime}=\left(N^{\prime}, V^{\prime}, \theta^{\prime}\right)$ are isomorphic, then $N \cong N^{\prime}$ and $V \cong V^{\prime}$. Hence, we may without loss of generality pose:

- $N^{\prime}=N=N_{x} \oplus N_{y}=\left(\bigoplus_{i} X_{i}^{\oplus m_{i}}\right) \oplus\left(\bigoplus_{j} Y_{j}^{\oplus n_{j}}\right)$ for some $m_{i}, n_{j} \in \mathbb{N}_{0}$.

- $V^{\prime}=V=\mathbb{k}^{t}$ for certain $t \in \mathbb{N}_{0}$.

Then the following is true: we have an isomorphism

$$
\left(N, V,\left(\Theta_{x}, \Theta_{y}\right)\right) \cong\left(N, V,\left(\Theta_{x}^{\prime}, \Theta_{y}^{\prime}\right)\right)
$$

in the category $\operatorname{Tri}(\mathrm{R})$ if any only if there exist automorphisms $\Psi_{x} \in \operatorname{Aut}_{\mathrm{S}_{x}}\left(N_{x}\right), \Psi_{y} \in$ Aut $_{\mathrm{S}_{y}}\left(N_{y}\right)$ and $\Phi \in \mathrm{GL}_{t}(\mathbb{k})$ such that

$$
\Theta_{x}^{\prime}=\bar{\Psi}_{x} \Theta_{x} \Phi^{-1} \quad \text { and } \quad \Theta_{y}^{\prime}=\bar{\Psi}_{y} \Theta_{y} \Phi^{-1},
$$

where $\bar{\Psi}_{x} \in \mathrm{GL}_{m}(\mathbb{k})$ (respectively $\bar{\Psi}_{y} \in \mathrm{GL}_{n}(\mathbb{k})$ ) is the induced automorphisms of $\bar{N}_{x} \cong \mathbb{k}^{m}$ (respectively $\bar{N}_{y} \cong \mathbb{k}^{n}$ ). The transformation rule (2.4) leads to the following problem of linear algebra (a matrix problem). 


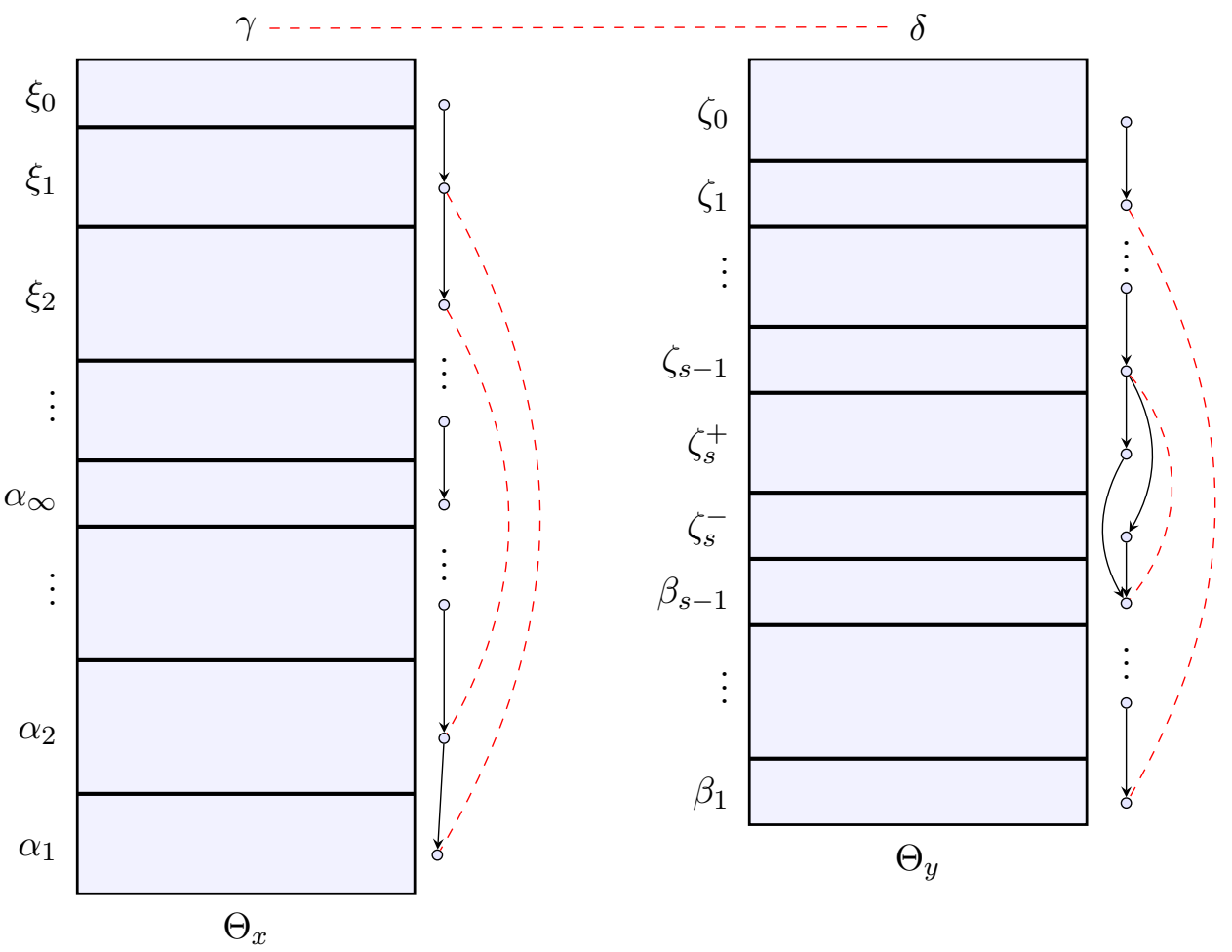

FiguRE 1. Matrix problem for the case $q=2 s$

- We have two matrices $\Theta_{x}$ and $\Theta_{y}$ over $\mathbb{k}$ with the same number of columns. The number of rows of $\Theta_{x}$ and $\Theta_{y}$ can be different. In particular, it can be zero for one of these matrices.

- Rows of $\Theta_{x}$ are divided into horizontal blocks indexed by elements of the linearly ordered set

$$
\mathfrak{E}_{x}=\left\{\xi_{0}<\xi_{1}<\cdots<\xi_{i}<\cdots<\alpha_{\infty}<\cdots<\alpha_{i}<\cdots<\alpha_{1}\right\} .
$$

The role of the ordering $<$ will be explained below.

- The block labeled by $\xi_{0}$ has $m_{0}$ rows, the block labeled by $\alpha_{\infty}$ has $m_{\infty}$ rows. The blocks labeled by $\xi_{i}$ and $\alpha_{i}$ both have $m_{i}$ rows. Thus, the matrix $\Theta_{x}$ has $m=m_{0}+m_{\infty}+2\left(m_{1}+\cdots+m_{i}+\ldots\right)$ rows.

- The row division of $\Theta_{y}$ depends on the parity of the parameter $q$.

- For $q=\infty$ the horizontal blocks of $\Theta_{y}$ are marked with the symbols of the linearly ordered set

$$
\mathfrak{E}_{y}=\mathfrak{E}_{y}^{\infty}=\left\{\zeta_{0}<\zeta_{1}<\cdots<\zeta_{j}<\cdots<\beta_{\infty}<\cdots<\beta_{j}<\cdots<\beta_{1}\right\},
$$

completely analogously as it is done for $\Theta_{x}$.

- For $q=2 s+1$, the labels are elements of the linearly ordered set

$$
\mathfrak{E}_{y}=\mathfrak{E}_{y}^{q}=\left\{\zeta_{0}<\zeta_{1}<\cdots<\zeta_{s}<\beta_{s}<\cdots<\beta_{1}\right\} .
$$

For any $1 \leq j \leq s$, the number of rows in blocks marked by $\zeta_{j}$ and $\beta_{j}$ is the same (and equal to $n_{j}$ ). 
- For $q=2 s$, the labels are elements of an (only partially!) ordered set

$$
\mathfrak{E}_{y}=\mathfrak{E}_{y}^{q}=\left\{\zeta_{0}<\zeta_{1}<\cdots<\zeta_{s-1}<\zeta_{s}^{+}, \zeta_{s}^{-}<\beta_{s-1}<\cdots<\beta_{1}\right\}
$$

as shown in Figure 1 (the elements $\zeta_{s}^{+}$and $\zeta_{s}^{-}$are incomparable). Again, the number of rows in blocks $\zeta_{j}$ and $\beta_{j}$ is the same for $1 \leq j \leq s-1$.

- We can perform any simultaneous elementary transformation of columns of $\Theta_{x}$ and $\Theta_{y}$.

- Transformations of rows of $\Theta_{x}$ are of three types.

- We can add any multiple of any row with lower weight to any row with higher weight.

- For any $i \in \mathbb{N}$ we can perform any simultaneous elementary transformation of rows within blocks marked by $\xi_{i}$ and $\alpha_{i}$.

- We can make any elementary transformation of rows in block $\xi_{0}$ or $\alpha_{\infty}$.

- The transformation rules for rows of $\Theta_{y}$ depend on the parity of $q$.

- Let us take the case $q$ is even (the most complicated one, see Figure 1).

* We can add any multiple of any row with lower weight to any row with higher weight.

* For any $1 \leq j \leq s-1$ we may perform any simultaneous elementary transformation of rows within blocks marked by $\zeta_{j}$ and $\beta_{j}$.

* We can make any (independent) elementary transformation of rows in the block $\zeta_{0}$ or $\zeta_{s}^{ \pm}$.

- For $q=\infty$, the transformation rules for $\Theta_{y}$ are analagous to those listed above for $\Theta_{x}$ (The matrix problem for this case will be studied in much detail in Subsection 3.1).

- For odd $q=2 s+1$, the transformation rules of $\Theta_{y}$ are the same as for $q=\infty$. The only difference between these cases lies in the absence of certain symbols in $\mathfrak{E}_{y}$.

4. For any $q \in \mathbb{N}_{\geq 2} \cup\{\infty\}$ the described matrix problem is an example of representations of a bunch of semi-chains. Tameness of the latter class of problems has been shown by Bondarenko in [6]. This implies the tameness of the category of triples Tri(R). Tameness of $\mathrm{CM}(\mathrm{R})$ follows from Theorem 1.19 .

Remark 2.2. Consider the following combinatorial data:

- The index set $I=\{x, y\}$.

- Let $\mathfrak{F}_{x}=\{\gamma\}, \mathfrak{F}_{y}=\{\delta\}, \mathfrak{F}=\mathfrak{F}_{x} \cup \mathfrak{F}_{y}$.

- Let $\mathfrak{E}_{x}$ and $\mathfrak{E}_{y}=\mathfrak{E}_{y}^{q}$ be as above, $\mathfrak{E}=\mathfrak{E}_{x} \cup \mathfrak{E}_{y}$.

- In the set $\mathfrak{B}=\mathfrak{E} \cup \mathfrak{F}$ consider the symmetric relation $\sim$ defined as follows:

$$
\begin{aligned}
& \gamma \sim \delta, \quad \xi_{i} \sim \alpha_{i} \quad \text { for } i \in \mathbb{N}^{*}, \\
& \zeta_{j} \sim \beta_{j} \quad \text { for } \begin{cases}1 \leq j \leq s & \text { if } q=2 s \text { or } q=2 s+1, \\
j \in \mathbb{N} & \text { if } q=\infty .\end{cases}
\end{aligned}
$$

The entire data (which is an example of a bunch of semi-chains) defines a certain bimodule category $\operatorname{Rep}(\mathfrak{B})$, see [13] or [11] for more details. The description of isomorphy classes of objects of $\operatorname{Rep}(\mathfrak{B})$ reduces precisely to the matrix problem described above. In our particular case, the category $\operatorname{Rep}(\mathfrak{B})$ admits the following intrinsic description: it is the comma category of the following diagram of categories and functors:

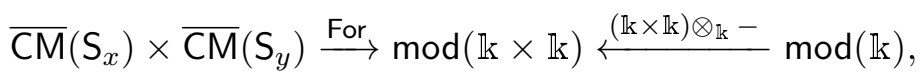


where $\overline{\mathrm{CM}}\left(\mathrm{S}_{x}\right)$ and $\overline{\mathrm{CM}}\left(\mathrm{S}_{y}\right)$ have been defined in Definition 1.25 (see also Lemma 1.26 for their explicit description) and For is the forgetful functor. According to Bondarenko [6], there are the following types of indecomposable objects in $\operatorname{Rep}(\mathfrak{B})$ : bands (continuous series) and (bispecial, special and usual) strings (discrete series). The precise combinatorics of the discrete series is rather complicated.

Definition 2.3. The forgetful functor $\mathbb{P}: \operatorname{Tri}(\mathrm{R}) \longrightarrow \operatorname{Rep}(\mathfrak{B})$ assigns assigns to a triple $\left(N, V,\left(\Theta_{x}, \Theta_{y}\right)\right)$ the pair of partitioned matrices $\left(\Theta_{x}, \Theta_{y}\right)$. To be more precise, we recall that

- $N=N_{x} \oplus N_{y}=\left(\bigoplus_{i} X_{i}^{\oplus m_{i}}\right) \oplus\left(\bigoplus_{j} Y_{j}^{\oplus n_{j}}\right)$ where $m_{i}, n_{j} \in \mathbb{N}_{0}$.

- $V=\mathbb{k}^{t}$ for some $t \in \mathbb{N}_{0}$.

- $\Theta_{x}: V=\mathbb{k}^{t} \longrightarrow \overline{\mathrm{S}} \otimes_{\mathrm{S}} N_{x} \cong \mathbb{k}^{m}$, $\Theta_{y}: V=\mathbb{k}^{t} \longrightarrow \overline{\mathrm{S}} \otimes_{\mathrm{S}} N_{y} \cong \mathbb{k}^{n}$ for certain $m, n \in \mathbb{N}_{0}$.

At this point, we have chosen bases for $\overline{\mathrm{S}} \otimes_{\mathrm{S}} N_{x}$ respectively $\overline{\mathrm{S}} \otimes_{\mathrm{S}} N_{y}$ which are induced by the distinguished generators of the indecomposable modules over $R_{x}$ and $R_{y}$ as in the body of the proof of Theorem 2.1. These generators correspond exactly to the elements of the bunch of semi-chains $\mathfrak{B}$ indexing the horizontal stripes of $\Theta_{x}$ and $\Theta_{y}$.

Remark 2.4. The functor $\mathbb{P}$ has the following properties.

(1) $\mathbb{P}$ is additive, full and preserves isomorphism classes of objects.

(2) The essential image of $\mathbb{P}$ consists of all pairs of matrices $(A, B)$ in $\operatorname{Rep}(\mathfrak{B})$ such that $A$ and $B$ have both full row rank and $\left(\begin{array}{c}A \\ B\end{array}\right)$ has full column rank.

(3) $\mathbb{P}$ is not faithful.

Remark 2.5. Let $\operatorname{char}(\mathbb{k})=2$. Then the simple curve singularities of type $A$ have to be redefined according to Remark 1.22. It follows that the equation of $\mathrm{P}_{\infty, 2 s}$ should be $\mathbb{k} \llbracket x, y, z \rrbracket /\left(x y, z\left(y^{s}-z\right)\right)$. Moreover, there are more singularities of type $\mathrm{P}_{\infty, 2 s+1}$, namely

$$
\mathrm{P}_{\infty, 2 s+1}^{t}:=\mathbb{k} \llbracket x, y, z \rrbracket /\left(x y, y^{2 s+1}+y^{s+t} z-z^{2}\right), \quad 1 \leq t \leq s-1 .
$$

Nevertheless, they are all tame and the proof of Theorem 2.1 applies literally to this case as well.

Remark 2.6. For any $q \in \mathbb{N} \cup\{\infty\}$ consider the hypersurface singularity

$$
\mathrm{T}=\mathrm{T}_{\infty, q+2}=\mathbb{k} \llbracket a, b \rrbracket /\left(b^{2}\left(a^{2}-b^{q}\right)\right) .
$$

Observe that $\mathrm{R}$ is an overring of $\mathrm{T}$ via the embedding

$$
\mathrm{T} \longrightarrow \mathrm{R}, \quad a \longmapsto x+v, \quad b \longmapsto y+u
$$

where $\mathrm{R}$ is the ring defined by (2.3). It was shown in [11, Section 11.1] that $\mathrm{CM}(\mathrm{T})$ has tame representation type (under the additional assumption $\operatorname{char}(\mathbb{k})=0$ ). This gives another argument that $\mathrm{CM}(\mathrm{R})$ (and hence $\mathrm{CM}(\mathrm{P})$ ) has either tame or discrete Cohen-Macaulay type. The latter case does also occur: if $q=1$, then $\mathrm{T}_{\infty 3}$ is representation tame whereas

$$
\mathrm{P}_{\infty 1}=\mathbb{k} \llbracket x, y, z \rrbracket /\left(x y, y-z^{2}\right) \cong \mathbb{k} \llbracket x, z \rrbracket /\left(x z^{2}\right)=: \mathrm{D}_{\infty}
$$

is representation discrete [9]. 


\section{Cohen-Macaulay modules over $\mathrm{P}_{\infty \infty}$ And $\mathrm{T}_{\infty \infty}$}

In this section we shall explain that the technique of matrix problems, introduced in the course of the proof of Theorem 2.1, leads to a completely explicit description of indecomposable Cohen-Macaulay modules over $\mathrm{P}=\mathrm{P}_{\infty \infty}=\mathbb{k} \llbracket x, y, z \rrbracket /\left(x y, z^{2}\right)$. Although $P$ is the "maximal degeneration" of the family (2.1), the combinatorics of the indecomposable objects in $\mathrm{CM}(\mathrm{P})$ are more transparent than for the less degenerate singularities $\mathrm{P}_{\infty, 2 r}$. The reason is that the underlying matrix problem has the type representations of bunches of chains and not of semi-chains as for $\mathrm{P}_{\infty, 2 r}$. Another motivation to study Cohen-Macaulay modules over $\mathrm{P}$ is that it allows to construct interesting examples of Cohen-Macaulay modules over the hypersurface singularity $\mathrm{T}=\mathrm{T}_{\infty \infty}=\mathbb{k} \llbracket a, b \rrbracket /\left(a^{2} b^{2}\right)$. Moreover, the explicit classification of indecomposable Cohen-Macaulay modules over $\mathrm{P}$ yields a classification for any curve singularity of type $\mathrm{P}_{\infty, 2 s+1}$ or $\mathrm{P}_{2 r+1,2 s+1}$.

Until the end of this section we keep the following notation:

- $\mathrm{R}=\mathbb{k} \llbracket x, y, u, v \rrbracket /\left(x y, y u, u v, v x, u^{2}, v^{2}\right)$ is the minimal overring of $\mathrm{P}$. The embed$\operatorname{ding} \mathrm{P} \longrightarrow \mathrm{R}$ sends $z$ to $u+v$.

- Let $\mathrm{S}=\mathrm{S}_{x} \times \mathrm{S}_{y}=\mathbb{k} \llbracket x, u \rrbracket /\left(u^{2}\right) \times \mathbb{k} \llbracket y, v \rrbracket /\left(v^{2}\right)$.

- For any $l \in \mathbb{N}$ we denote $X_{l}=\left(u, x^{l}\right)_{\mathrm{S}}$ and $Y_{l}=\left(v, y^{l}\right)_{\mathrm{s}}$. Next, we pose $X_{0}=\mathrm{S}_{x}$, $Y_{0}=\mathrm{S}_{y}, X_{\infty}=(u)_{\mathrm{S}}$ and $Y_{\infty}=(v)_{\mathrm{S}}$.

- Let $\mathbf{Q}=\mathbb{k}((x))[u] /\left(u^{2}\right) \times \mathbb{k}((y))[v] /\left(v^{2}\right)$.

- Denote $\mathfrak{m}=(x, y, u, v)_{\mathrm{R}}$. Recall that $\mathfrak{m}=\operatorname{ann}_{\mathrm{R}}(\mathrm{S} / \mathrm{R})=\operatorname{rad}(\mathrm{S})$.

Observe that $\mathrm{Q}$ is the common total ring of fractions of $\mathrm{P}, \mathrm{R}$ and $\mathrm{S}$. In particular, we have the following equalities in $\mathrm{Q}$ :

$$
u=\frac{x z}{x+y}, \quad v=\frac{y z}{x+y}, \quad e_{x}:=(1,0)=\frac{x}{x+y} \quad \text { and } \quad e_{y}:=(0,1)=\frac{y}{x+y} .
$$

Next, note that $\mathrm{R}$ is also an overring of $\mathrm{T}$. Indeed, we have an injective ring homomorphism $\jmath: \mathrm{T} \longrightarrow \mathrm{R}$ given by $a \mapsto x+v$ and $b \mapsto y+u$. It is also not difficult to see that $\jmath$ induces an isomorphism of the total rings of fractions $Q(T) \longrightarrow Q(R)=Q$. Summing up, we have the following diagram of categories and functors:

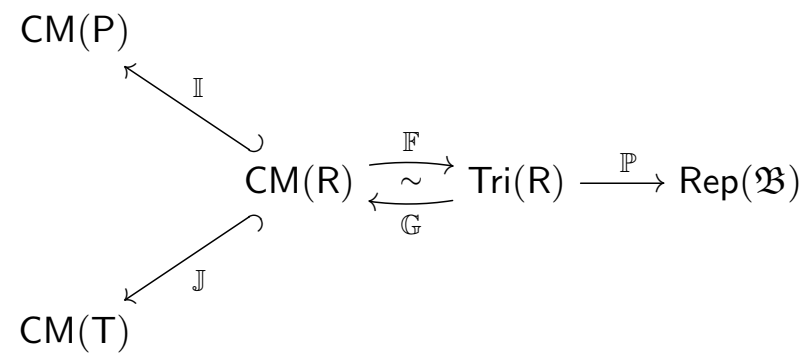

- $\mathbb{I}$ and $\mathbb{J}$ denote restriction functors. According to Proposition 1.9 , they are both fully faithful. Moreover, by Theorem 1.11 Ind $(C M(P))=\{P\} \cup \operatorname{Ind}(C M(R))$.

- $\mathbb{F}$ and $\mathbb{G}$ are quasi-inverse equivalences of categories from Theorem 1.19 .

- The functor $\mathbb{P}$ assigns to a triple $\left(N, V,\left(\Theta_{x}, \Theta_{y}\right)\right)$ the pair of matrices $\left(\Theta_{x}, \Theta_{y}\right)$, whose rows are equipped with some additional "weights". $\mathbb{P}$ preserves isomorphy classes of objects as well as their indecomposability. However, $\mathbb{P}$ is not essentially surjective because $\Theta_{x}$ and $\Theta_{y}$ obey some additional constraints, see (3.3). 


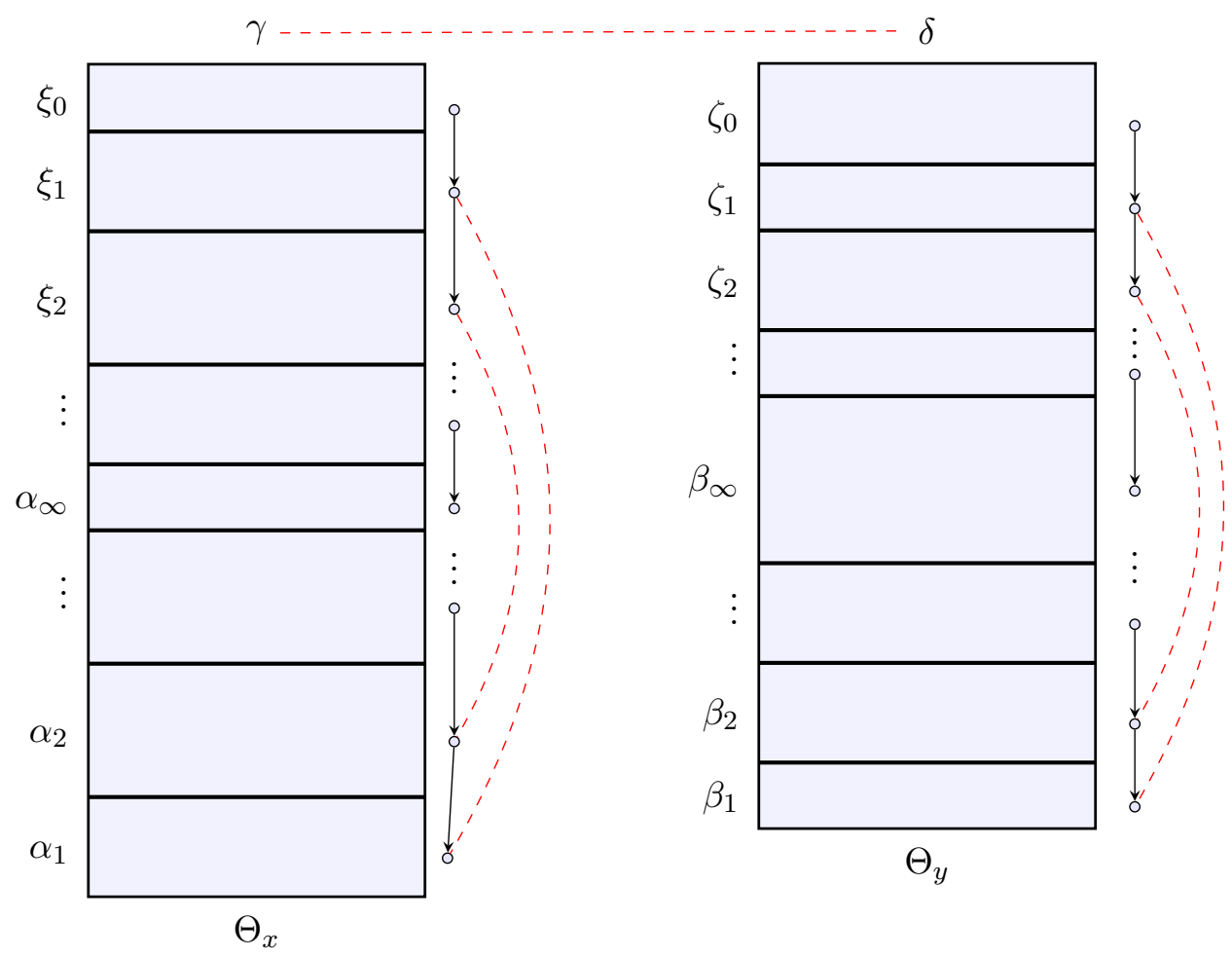

FiguRE 2. Matrix problem for the case $q=\infty$

The goal of this section is to show how one can translate the combinatorics of indecomposable objects of $\operatorname{Rep}(\mathfrak{B})$ into an explicit description of indecomposable objects of $\mathrm{CM}(\mathrm{P})$ and $\mathrm{CM}(\mathrm{T})$.

3.1. Indecomposable objects of $\operatorname{Rep}(\mathfrak{B})$. According to Theorem 2.1, the matrix problem corresponding to a description of isomorphy classes of objects in $\operatorname{Rep}(\mathfrak{B})$ is as follows.

We are given two matrices $\Theta_{x}$ and $\Theta_{y}$ as depicted in Figure 2 with entries from an algebraically closed field $\mathbb{k}$ and the same number of columns. The rows of $\Theta_{x}$ and $\Theta_{y}$ are both divided into horizontal blocks. Any two horizontal blocks in $\Theta_{x}$ (respectively $\Theta_{y}$ ) connected by a dotted line have the same number of rows.

Transformation rules. The following transformations of columns and rows of $\Theta_{x}$ and $\Theta_{y}$ are admissible:

- any simultaneous elementary transformation of columns of $\Theta_{x}$ and $\Theta_{y}$.

- addition of any multiple of any row of $\Theta_{x}$ (respectively, $\Theta_{y}$ ) with lower weight to any row of $\Theta_{x}$ (respectively, $\Theta_{y}$ ) with higher weight.

- any simultaneous elementary transformation of rows within horizontal blocks of $\Theta_{x}$ (respectively, $\Theta_{y}$ ) connected by a dotted line.

- any elementary transformation of rows in the horizontal block of $\Theta_{x}$ (respectively, $\left.\Theta_{y}\right)$ which is not connected to any other block by a dotted line. 
Additionally, there are the following regularity constraints on $\Theta_{x}$ and $\Theta_{y}$ :

$$
\begin{aligned}
& \Theta_{x} \text { and } \Theta_{y} \text { have both full row rank, } \\
& \text { the matrix }\left(\begin{array}{l}
\Theta_{x} \\
\Theta_{y}
\end{array}\right) \text { has full column rank. }
\end{aligned}
$$

In this subsection we are going to apply Bondarenko's result [6] to describe the canonical forms of the matrix problem above.

Definition 3.1. Consider the following data:

- The index set $I=\{x, y\}$.

- The set of column symbols $\mathfrak{F}=\mathfrak{F}_{x} \cup \mathfrak{F}_{y}$, where $\mathfrak{F}_{x}=\{\gamma\}, \mathfrak{F}_{y}=\{\delta\}$.

- The set of row symbols $\mathfrak{E}=\mathfrak{E}_{x} \cup \mathfrak{E}_{y}$, where

$$
\begin{aligned}
& \mathfrak{E}_{x}=\left\{\xi_{0}<\xi_{1}<\cdots<\xi_{i}<\cdots<\alpha_{\infty}<\cdots<\alpha_{i}<\cdots<\alpha_{1}\right\}, \\
& \mathfrak{E}_{y}=\left\{\zeta_{0}<\zeta_{1}<\cdots<\zeta_{j}<\cdots<\beta_{\infty}<\cdots<\beta_{j}<\cdots<\beta_{1}\right\} .
\end{aligned}
$$

- The set $\mathfrak{B}=\mathfrak{E} \cup \mathfrak{F}$ is equipped with a symmetric relation $\sim$ defined as follows:

$$
\gamma \sim \delta, \quad \xi_{l} \sim \alpha_{l} \quad \text { and } \quad \zeta_{l} \sim \beta_{l} \quad \text { for } l \in \mathbb{N}^{*} .
$$

In addition, we introduce another symmetric relation - on the set $\mathfrak{B}$ as follows:

$$
\gamma-\epsilon_{x} \text { for any } \epsilon_{x} \in \mathfrak{E}_{x} \text { and } \delta-\epsilon_{y} \text { for any } \epsilon_{y} \in \mathfrak{E}_{y} .
$$

The problem to classify the indecomposable objects of the category $\operatorname{Rep}(\mathfrak{B})$ up to isomorphism is exactly the matrix problem above without the "regularity conditions" (3.3).

Now, we define strings and bands of the bunch of chains $\mathfrak{B}$. They describe the invariants of the indecomposable representations in $\operatorname{Rep}(\mathfrak{B})$.

Definition 3.2. Let $\mathfrak{B}$ be the bunch of chains from Definition 3.1.

(1) A full word $w$ of $\mathfrak{B}$ is a sequence

$$
w=\chi_{1} \rho_{1} \chi_{2} \rho_{2} \ldots \chi_{n-1} \rho_{n-1} \chi_{n}
$$

of symbols $\chi_{k} \in \mathfrak{B}$ and relations $\rho_{k} \in\{\sim,-\}$ subject to the following conditions:

- the relation $\chi_{k} \rho_{k} \chi_{k+1}$ holds in $\mathfrak{B}$ for $1 \leq k \leq n-1$.

- the sequence of relations alternates, i.e. $\rho_{k} \neq \rho_{k+1}$ for $1 \leq k \leq n-2$.

- either $\chi_{1} \in\left\{\xi_{0}, \alpha_{\infty}, \zeta_{0}, \beta_{\infty}\right\}$ or $\rho_{1}$ is $\sim$.

- either $\chi_{n} \in\left\{\xi_{0}, \alpha_{\infty}, \zeta_{0}, \beta_{\infty}\right\}$ or $\rho_{n-1}$ is $\sim$.

(2) The opposite word of a full word $w$ as above is defined by

$$
w^{o}=\chi_{n} \rho_{n-1} \chi_{n-1} \rho_{n-2} \cdots \chi_{2} \rho_{1} \chi_{1} .
$$

(3) A string datum of $\mathfrak{B}$ is given by any full word $w$.

(4) Two string data $w$ and $w^{\prime}$ are equivalent if and only if $w^{\prime}=w$ or $w^{\prime}=w^{o}$.

Definition 3.3. Let $\mathfrak{B}$ be the bunch of chains from Definition 3.1 .

(1) A cyclic word $w$ is given by a full word $w=\chi_{1} \rho_{1} \chi_{2} \ldots \chi_{n-1} \rho_{n-1} \chi_{n}$ and an additional relation $\rho_{n}$ equal to - such that the following additional conditions are satisfied:

- $\chi_{n} \rho_{n} \chi_{1}$ holds in $\mathfrak{B}$.

- $\rho_{1}$ and $\rho_{n-1}$ are equal to $\sim$.

(2) The opposite of a cyclic word as above is given by the full word $w^{o}$ together with the additional relation $\rho_{n}=-$. 
(3) For any even integer $k \in \mathbb{Z} / n \mathbb{Z}$ the $k$-th shift of $w$ is defined by

$$
w^{[k]}=\chi_{k+1} \rho_{k+1} \chi_{k+2} \rho_{k+2} \ldots \chi_{k+n-1} \rho_{k+n-1} \chi_{k+n} \rho_{k+n},
$$

where the indices are considered modulo $n$.

(4) A cyclic word $w$ is periodic if $w=w^{[k]}$ for some even non-trivial shift $k \in(\mathbb{Z} / n \mathbb{Z})^{*}$.

(5) A band datum $(w, m, \lambda)$ of $\mathfrak{B}$ consists of a non-periodic cyclic word $w$, a multiplicity parameter $m \in \mathbb{N}$ and a continuous parameter $\lambda \in \mathbb{k}^{*}$.

(6) Two band data $(w, m, \lambda)$ and $\left(w^{\prime}, m^{\prime}, \lambda^{\prime}\right)$ are equivalent if and only if both words $w$ and $w^{\prime}$ have the same length $n$ and if $\left(w^{\prime}, m^{\prime}, \lambda^{\prime}\right)$ is given by $\left(w^{o}, m, \lambda\right),\left(w^{[4 l]}, m, \lambda\right)$ or $\left(w^{[4 l+2]}, m, \lambda^{-1}\right)$ for some $l \in \mathbb{Z} / n \mathbb{Z}$.

The above definitions are motivated by the following result of Bondarenko [6].

Theorem 3.4. There is a bijection between the equivalence classes of string and band data of the bunch of chains $\mathfrak{B}$ and the isomorphism classes of indecomposable objects in the category $\operatorname{Rep}(\mathfrak{B})$.

Now we explain Bondarenko's construction of indecomposable objects in $\operatorname{Rep}(\mathfrak{B})$ corresponding to a string or band datum.

1. Let $w$ be a string datum of $\mathfrak{B}$. The corresponding object $\Theta(w)$ of $\operatorname{Rep}(\mathfrak{B})$ is given by a pair of matrices $\Theta_{x}(w)$ and $\Theta_{y}(w)$ defined as follows:

(1) Let $t$ be the number of times the symbol $\gamma$ (or $\delta$ ) occurs as a letter in $w$. Then both matrices $\Theta_{x}(w)$ and $\Theta_{y}(w)$ have $t$ columns.

(2) For each $\epsilon \in \mathfrak{E}$, let $m_{\epsilon}$ be the number of times the symbol $\epsilon$ occurs as a letter in $w$. Then the horizontal block $\epsilon$ in $\Theta_{x}(w)$ (respectively $\left.\Theta_{y}(w)\right)$ has $m_{\epsilon}$ rows.

(3) Next, we assign to every letter $\chi_{k}$ in $w$ the number of times the letter $\chi_{k}$ occurred in the subword $\chi_{1} \rho_{1} \ldots \rho_{k-1} \chi_{k}$. In other words, we number every letter in $w$ by the time it occurs in $w$.

(4) Every appearance of the relation - in $w$ contributes to a non-zero entry in $\Theta(w)$ in the following way. Let $\epsilon-\nu$ or $\nu-\epsilon$ be a subword in $w$ such that $\epsilon \in \mathfrak{E}$ and $\nu \in \mathfrak{F}$. Let $i$ be the occurrence number of $\epsilon$ and $j$ be the occurrence number of $\nu$. We fill the entries of $\Theta_{x}$ respectively $\Theta_{y}$ according to the following rule.

- If $\nu=\gamma$ (respectively $\nu=\delta)$, the $(i, j)$-th entry of the $\epsilon$-th horizontal block of $\Theta_{x}$ (respectively $\Theta_{y}$ ) is set to be 1 . This rule is applied for every relation - in $w$.

- All remaining entries of $\Theta_{x}$ and $\Theta_{y}$ are set to be 0 .

2. Let $(w, m, \lambda)$ be a band datum. The corresponding object $\Theta(w, m, \lambda)$ of $\operatorname{Rep}(\mathfrak{B})$ is given by a pair of matrices $\left(\Theta_{x}(w, m, \lambda), \Theta_{y}(w, m, \lambda)\right)$ defined as follows.

(1) First construct the canonical form $\Theta(w)$, where $w$ is viewed as a string parameter.

(2) Replace any zero entry in $\Theta_{x}(w)$ (respectively $\Theta_{y}(w)$ ) by the zero matrix of size $m$ and any identity entry in $\Theta_{x}(w)$ (respectively $\Theta_{y}(w)$ ) by the identity matrix $I$ of size $m$.

(3) We consider the last relation $\rho_{n}=-$ in $w$. Then $\chi_{n} \rho_{n} \chi_{1}$ in $\mathfrak{B}$ and either $\chi_{1}$ or $\chi_{n} \in \mathfrak{E}$. Replace the zero matrix at the intersection of the first $m$ rows of the horizontal block indexed by $\chi_{1}$ (respectively $\chi_{n}$ ) and its last $m$ columns by the Jordan block $J_{m}(\lambda)$ with eigenvalue $\lambda$ and size $m$. 
Remark 3.5. Let $\Theta$ be the object of $\operatorname{Rep}(\mathfrak{B})$ associated to a string or band datum. It is straightforward to show, that $\Theta$ satisfies the regularity constraints (3.3) if and only if the following two conditions are satisfied:

- either $w$ is cyclic or $w$ begins and ends with symbols from $\mathfrak{F}$.

- $w$ is neither equal to $\gamma \sim \delta$ nor equal to $\delta \sim \gamma$.

Next, we give some examples of canonical forms of band and string data.

Example 3.6. Consider a band datum $(w, m, \lambda)$ where $w$ is given by the following cyclic word:

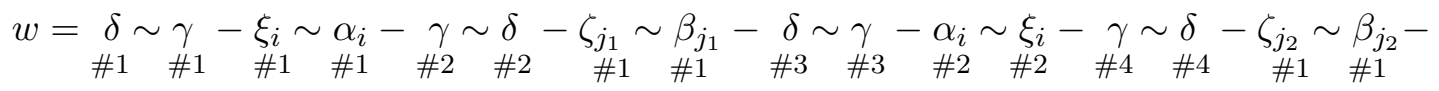

Then the corresponding canonical forms $\left(\Theta_{x}(w, m, \lambda), \Theta_{y}(w, m, \lambda)\right)$ are the following:

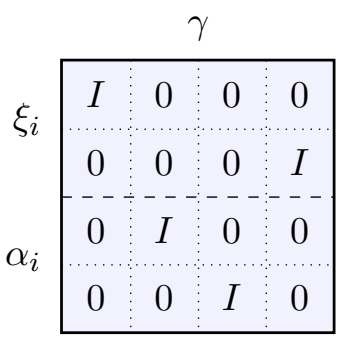

\begin{tabular}{|cccc:c|}
\multicolumn{1}{c}{$\delta$} \\
\hline 0 & $I$ & 0 & 0 & $\zeta_{j_{1}}$ \\
\hdashline 0 & 0 & 0 & $I$ & $\zeta_{j_{2}}$ \\
\hdashline 0 & 0 & $I$ & 0 & $\beta_{j_{1}}$ \\
\hdashline$J$ & 0 & 0 & 0 & $\beta_{j_{2}}$
\end{tabular}

Here, $I$ is the identity matrix of size $m$ and $J=J_{m}(\lambda)$ is the Jordan block of size $m$ with eigenvalue $\lambda \in \mathbb{k}^{*}$.

Example 3.7. Consider the string datum given by the word

$$
w=\delta \sim \gamma-\xi_{i} \sim \alpha_{i}-\gamma \sim \delta-\zeta_{j} \sim \beta_{j}-\delta \sim \gamma .
$$

Then the corresponding canonical forms $\left(\Theta_{x}(w), \Theta_{y}(w)\right)$ are the following:

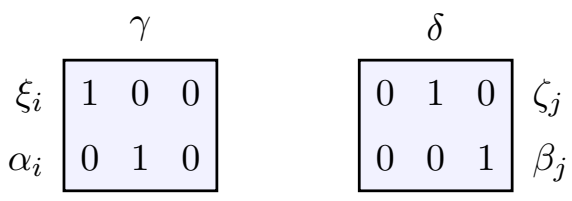

Example 3.8. Consider the string datum given by the word

$$
w=\xi_{0}-\gamma \sim \delta-\zeta_{j_{1}} \sim \beta_{j_{1}}-\delta \sim \gamma-\xi_{i} \sim \alpha_{i}-\gamma \sim \delta-\beta_{j_{2}} \sim \zeta_{j_{2}}-\delta \sim \gamma
$$

Then the corresponding canonical forms $\left(\Theta_{x}(w), \Theta_{y}(w)\right)$ are the following:

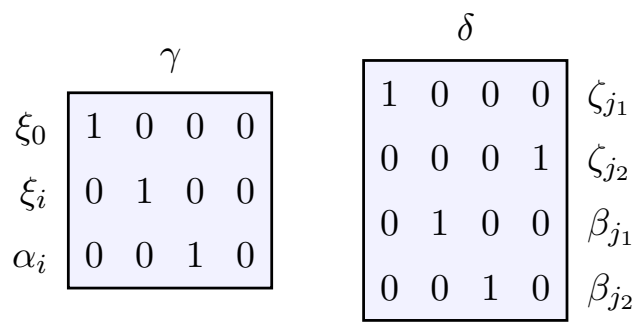

Example 3.9. Consider the string datum given by the word

$$
w=\alpha_{\infty}-\gamma \sim \delta-\beta_{j} \sim \zeta_{j}-\delta \sim \gamma-\xi_{i} \sim \alpha_{i}-\gamma \sim \delta-\beta_{\infty} .
$$

Then the corresponding canonical forms $\left(\left(\Theta_{x}(w), \Theta_{y}(w)\right)\right.$ are the following: 


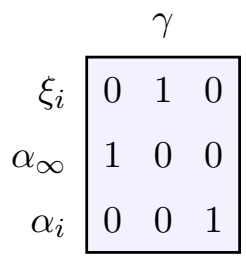

\begin{tabular}{|lll|l}
\multicolumn{3}{c}{$\delta$} & \multicolumn{1}{c}{} \\
\cline { 1 - 2 } 0 & 1 & 0 & $\zeta_{j}$ \\
0 & 0 & 1 & $\beta_{\infty}$ \\
1 & 0 & 0 & $\beta_{j}$
\end{tabular}

3.2. Indecomposable Cohen-Macaulay modules over $\mathrm{P}_{\infty \infty}$. Consider an object $\mathrm{T}=\left(N, V,\left(\Theta_{x}, \Theta_{y}\right)\right)$ of the category $\operatorname{Tr}(\mathrm{R})$. Then we may assume that

- $N=X_{i_{1}} \oplus \cdots \oplus X_{i_{k}} \oplus Y_{j_{1}} \oplus \cdots \oplus Y_{j_{l}} \subseteq \mathrm{S}^{k+l}$ for certain indices $i_{1}, \ldots, i_{k}, j_{1}, \ldots, j_{l} \in$ $\mathbb{N}_{0} \cup\{\infty\}$, see the beginning of Section 3 for the definition of S-modules $X_{i}$ and $Y_{i}$ for $i \in \mathbb{N}_{0} \cup\{\infty\}$.

- $V=\mathbb{k}^{t}$ for some $t \in \mathbb{N}_{0}$.

According to Theorem 1.19, the corresponding Cohen-Macaulay R-module $M=\mathbb{G}(\mathrm{T})$ is determined by the following commutative diagram in $\bmod (R)$ :

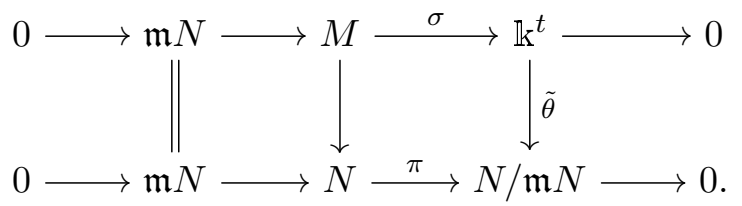

Lemma 3.10. Let $\left\{e_{1}, \ldots, e_{t}\right\}$ be the standard basis of $\mathbb{k}^{t}$. For any $1 \leq i \leq t$ choose $w_{i} \in N$ such that $\pi\left(w_{i}\right)=\tilde{\theta}\left(e_{i}\right)$. Then we have:

$$
M=\left\langle w_{1}, \ldots, w_{t}\right\rangle_{\mathrm{R}} \subseteq \mathrm{S}^{k+l}
$$

and $t$ is the minimal number of generators of $M$.

Proof. By definition of $M$, for any $1 \leq i \leq t$ we have: $w_{i} \in M$. Next, the induced map $\bar{\sigma}: M / \mathfrak{m} M \longrightarrow \mathbb{k}^{t}$ is an isomorphism (see the proof of [11, Theorem 12.5]) and $\bar{\sigma}\left(w_{i}\right)=e_{i}$. Hence, $\left\{\bar{w}_{1}, \ldots, \bar{w}_{t}\right\}$ is a basis of $M / \mathfrak{m} M$ and $(3.5)$ follows from Nakayama's Lemma.

Lemma 3.11. Let $\mathrm{T}=\left(N, \mathbb{k}^{t},\left(\Theta_{x}, \Theta_{y}\right)\right)$ be an indecomposable object of $\operatorname{Tri}(\mathrm{R})$ as above and $M=\mathbb{G}(\mathrm{T})=\left\langle w_{1}, \ldots, w_{t}\right\rangle_{\mathrm{R}} \subseteq \mathrm{S}^{k+l}$. Then the following results are true.

(1) We have either

- $\mathrm{T} \cong(\mathrm{S}, \mathbb{k},((1),(1)))$ (in this case $M \cong \mathrm{R})$, or

- $\mathbb{I}(M)=\left\langle w_{1}, \ldots, w_{t}\right\rangle_{\mathrm{P}} \subseteq \mathrm{S}^{k+l}$.

In both cases we have:

$$
\mathbb{J}(M)=\left\langle w_{1}, \ldots, w_{t}, v w_{1}, \ldots, v w_{t}, u w_{1}, \ldots, u w_{t}\right\rangle_{\mathrm{T}} \subseteq \mathrm{S}^{k+l} .
$$

(2) The Cohen-Macaulay module $M$, respectively $\mathbb{I}(M)$ and $\mathbb{J}(M)$, is locally free on the punctured spectrum of $\mathrm{R}$, respectively $\mathrm{P}$ and $\mathrm{T}$, if and only if $N$ contains no direct summands isomorphic to $X_{\infty}$ or $Y_{\infty}$.

Proof. (1) The statement about $\mathbb{I}(M)$ is a corollary of Proposition 1.13. The statement about $\mathbb{J}(M)$ follows from the fact that $\mathbb{J}(\mathrm{T})=\langle 1, u, v\rangle_{\mathrm{R}} \subset Q$ in $\mathrm{R}-\bmod$.

(2) The Cohen-Macaulay module $M$ is locally free on the punctured spectrum of $\mathrm{R}$ if and only $M \cong \mathrm{S} \otimes_{\mathrm{R}} M$ is locally free on the punctured spectrum of $\mathrm{S}$, see Remark 1.15. The latter is equivalent that $N$ contains no direct summands isomorphic to $X_{\infty}$ and $Y_{\infty}$. Since the rational envelopes of $M, \mathbb{I}(M)$ and $\mathbb{J}(M)$ are the same, the result follows. 
Now we are ready to state the final classification of the indecomposable Cohen-Macaulay $\mathrm{P}-$ modules. For any $l \in \mathbb{N}$ introduce the letters $\mathbf{x}_{l}^{ \pm}$and $\mathbf{y}_{l}^{ \pm}$as well as $\mathbf{x}_{0}, \mathbf{x}_{\infty}, \mathbf{y}_{0}$ and $\mathbf{y}_{\infty}$.

Definition 3.12. Band modules $B=B(\omega, m, \lambda)$ have the following combinatorics:

- $\omega=\mathbf{x}_{i_{1}}^{\sigma_{1}} \mathbf{y}_{j_{1}}^{\tau_{1}} \ldots \mathbf{x}_{i_{n}}^{\sigma_{n}} \mathbf{y}_{j_{n}}^{\tau_{n}}$ is a non-periodic word, where $\sigma_{k}, \tau_{k} \in\{+,-\}$ and $i_{k}, j_{k} \in \mathbb{N}$ for $1 \leq k \leq n$.

- $m \in \mathbb{N}$ and $\lambda \in \mathbb{k}^{*}$.

Consider the following Cohen-Macaulay S-module

$$
N:=X_{i_{1}}^{\oplus m} \oplus Y_{j_{1}}^{\oplus m} \oplus \cdots \oplus X_{i_{n}}^{\oplus m} \oplus Y_{j_{n}}^{\oplus m} \subseteq \mathrm{S}^{2 m n} .
$$

Then $B$ is the following $\mathrm{R}$-submodule of $N$ :

$$
B:=\left[\left(\begin{array}{c}
f_{i_{1} I}^{\prime \prime} I \\
g_{j_{1}}^{\prime} I \\
0 \\
0 \\
\vdots \\
0 \\
0
\end{array}\right),\left(\begin{array}{c}
0 \\
g_{j_{1}}^{\prime \prime} I \\
f_{i_{2}}^{\prime} I \\
0 \\
\vdots \\
0 \\
0
\end{array}\right), \ldots, \quad\left(\begin{array}{c}
0 \\
0 \\
0 \\
0 \\
\vdots \\
f_{i_{n}}^{\prime \prime} I \\
g_{j_{n}}^{\prime} I
\end{array}\right), \quad\left(\begin{array}{c}
f_{i_{1}}^{\prime} J \\
0 \\
0 \\
0 \\
\vdots \\
0 \\
g_{j_{n}}^{\prime \prime} I
\end{array}\right)\right]_{\mathrm{R}} \subseteq N,
$$

where for any $1 \leq k \leq n$ the elements $f_{i_{k}}^{\prime}, f_{i_{k}}^{\prime \prime}, g_{j_{k}}^{\prime} g_{j_{k}}^{\prime \prime}$ are defined by the tables:

\begin{tabular}{|c|c|c|}
\hline$\sigma_{k}$ & $f_{i_{k}}^{\prime}$ & $f_{i_{k}}^{\prime \prime}$ \\
\hline+ & $u$ & $x^{i_{k}}$ \\
- & $x^{i_{k}}$ & $u$ \\
\hline
\end{tabular}

\begin{tabular}{|c|c|c|}
\hline$\tau_{k}$ & $g_{j_{k}}^{\prime}$ & $g_{j_{k}}^{\prime \prime}$ \\
\hline+ & $v$ & $y^{j_{k}}$ \\
- & $y^{j_{k}}$ & $v$ \\
\hline
\end{tabular}

Definition 3.13. A string module $S=S(\omega)$ is defined by a word $\omega$ of the following form. $\omega$ has a beginning, an intermediate part and an end. The beginning as well as the end may consist of zero, one or two letters. The following table lists all possible beginnings and ends for $\omega$ (any beginning from the first column can match any ending from the last column):

\begin{tabular}{|r|l|l|}
\hline$\hat{\mathbf{z}}_{i_{0}} \check{\mathbf{z}}_{j_{0}}^{\tau_{0}}$ & intermediate part & $\hat{\mathbf{z}}_{i_{n}}^{\sigma_{n}} \check{\mathbf{z}}_{j_{n}}$ \\
\hline \hline void & & void \\
$\mathbf{y}_{0}$ & & $\mathbf{x}_{0}$ \\
$\mathbf{y}_{\infty}$ & $\mathbf{x}_{i_{1}}^{\sigma_{1}} \mathbf{y}_{j_{1}}^{\tau_{1}} \ldots \mathbf{x}_{i_{n-1}}^{\sigma_{n-1}} \mathbf{y}_{j_{n-1}}^{\tau_{n-1}}$ & $\mathbf{x}_{\infty}$ \\
$\mathbf{y}_{j_{0}}^{\tau_{0}}$ & $\mathbf{x}_{i_{n}}^{\sigma_{n}}$ \\
$\mathbf{x}_{0} \mathbf{y}_{j_{0}}^{\tau_{0}}$ & & $\mathbf{x}_{i_{n}}^{\sigma_{n}} \mathbf{y}_{0}$ \\
$\mathbf{x}_{\infty} \mathbf{y}_{j_{0}}^{\tau_{0}}$ & & $\mathbf{x}_{i_{n}}^{\sigma_{n}} \mathbf{y}_{\infty}$ \\
\hline
\end{tabular}

where

- $n \in \mathbb{N}$. For $n=1$ the intermediate part of $\omega$ is void.

- For any $0 \leq k \leq n$ we have: $i_{k}, j_{k} \in \mathbb{N}$ and $\sigma_{k}, \tau_{k} \in\{+,-\}$.

In other words, a string word $\omega$ is given by an alternating sequence of letters $\mathbf{x}_{i}$ or $\mathbf{y}_{j}$ such that a letter of the form $\mathbf{x}_{0}, \mathbf{x}_{\infty}, \mathbf{y}_{0}$ or $\mathbf{y}_{\infty}$ may only occur as the first or last letter of $\omega$. 
Consider the Cohen-Macaulay S-module

$$
N=\hat{Z}_{i_{0}} \oplus \check{Z}_{j_{0}} \oplus X_{i_{1}} \oplus Y_{j_{1}} \oplus \cdots \oplus X_{i_{n-1}} \oplus Y_{j_{n-1}} \oplus \hat{Z}_{i_{n}} \oplus \check{Z}_{j_{n}}
$$

where for each $i \in\left\{i_{0}, i_{n}\right\}$ and each $j \in\left\{j_{0}, j_{n}\right\}$, we set

$$
\hat{Z}_{i}=\left\{\begin{array}{ll}
X_{i} & \text { if } \mathbf{z}_{i}=\mathbf{x}_{i}, \\
0 & \text { if } \mathbf{z}_{i} \text { is void. }
\end{array} \quad \check{Z}_{j}= \begin{cases}Y_{j} & \text { if } \mathbf{z}_{j}=\mathbf{y}_{j}, \\
0 & \text { if } \mathbf{z}_{j} \text { is void }\end{cases}\right.
$$

Then $S=S(\omega)$ is the following R-submodule of $N$ :

$$
S:=\left[\left(\begin{array}{c}
f_{i_{0}}^{\prime \prime} \\
g_{j_{0}}^{\prime} \\
0 \\
\vdots \\
0 \\
0 \\
0
\end{array}\right),\left(\begin{array}{c}
0 \\
g_{j_{0}}^{\prime \prime} \\
f_{i_{1}}^{\prime} \\
\vdots \\
0 \\
0 \\
0
\end{array}\right), \ldots, \quad\left(\begin{array}{c}
0 \\
0 \\
0 \\
\vdots \\
g_{j_{n-1}}^{\prime \prime} \\
f_{i_{n}}^{\prime} \\
0
\end{array}\right),\left(\begin{array}{c}
0 \\
0 \\
0 \\
\vdots \\
0 \\
f_{i_{n}}^{\prime \prime} \\
g_{j_{n}}^{\prime}
\end{array}\right)\right]_{\mathrm{R}} \subseteq N
$$

where for any $1 \leq k \leq n$, the elements $f_{i_{k}}^{\prime}, f_{i_{k}}^{\prime \prime}, g_{j_{k}}^{\prime}$ and $g_{j_{k}}^{\prime \prime}$ are defined by the tables:

\begin{tabular}{|c|c|c|}
\hline$\sigma_{k}$ & $f_{i_{k}}^{\prime}$ & $f_{i_{k}}^{\prime \prime}$ \\
\hline+ & $u x$ & $x^{i_{k}+1}$ \\
- & $x^{i_{k}+1}$ & $u x$ \\
\hline
\end{tabular}

\begin{tabular}{|c|c|c|}
\hline$\tau_{k}$ & $g_{j_{k}}^{\prime}$ & $g_{j_{k}}^{\prime \prime}$ \\
\hline+ & $v y$ & $y^{j_{k}+1}$ \\
- & $y^{j_{k}+1}$ & $v y$ \\
\hline
\end{tabular}

The remaining entries are defined as follows:

\begin{tabular}{|l|c|}
\hline & $f_{i_{0}}^{\prime \prime}$ \\
\hline$i_{0}=0$ & $x$ \\
$i_{0}=\infty$ & $u x$ \\
$\hat{\mathbf{z}}_{i_{0}}$ is void & 0 \\
\hline
\end{tabular}

\begin{tabular}{|l|c|}
\hline & $g_{j_{n}}^{\prime}$ \\
\hline$j_{n}=0$ & $y$ \\
$j_{n}=\infty$ & $v y$ \\
$\check{\mathbf{z}}_{j_{n}}$ is void & 0 \\
\hline
\end{tabular}

\begin{tabular}{|c|c|c|c|}
\hline & $\sigma_{n}$ & $f_{i_{n}}^{\prime}$ & $f_{i_{n}}^{\prime \prime}$ \\
\hline$i_{n}=0$ & & $x$ & 0 \\
$i_{n} \in \mathbb{N}$ & + & $u x$ & $x^{j_{n}+1}$ \\
$i_{n} \in \mathbb{N}$ & - & $x^{j_{n}+1}$ & $u x$ \\
$i_{n}=\infty$ & & $u x$ & 0 \\
\hline
\end{tabular}

\begin{tabular}{|c|c|c|c|}
\hline & $\tau_{0}$ & $g_{j_{0}}^{\prime}$ & $g_{j_{0}}^{\prime \prime}$ \\
\hline$j_{0}=0$ & & 0 & $y$ \\
$j_{0} \in \mathbb{N}$ & + & $v y$ & $y^{j_{0}+1}$ \\
$j_{0} \in \mathbb{N}$ & - & $y^{j_{0}+1}$ & $v y$ \\
$j_{0}=\infty$ & & 0 & $v y$ \\
\hline
\end{tabular}

Remark 3.14. If the string parameter $\omega$ contains neither $\mathbf{x}_{0}$ nor $\mathbf{y}_{0}$, there is a better presentation of the module $S(\omega)$ : we divide all entries of type $f_{i}^{\prime}$ or $f_{i}^{\prime \prime}$ of $S(\omega)$ by $x$ and all entries of type $g_{j}^{\prime}$ or $g_{j}^{\prime \prime}$ of $S(\omega)$ by $y$. 
Remark 3.15. Any string module $S$ in $(3.8)$ has a more compact presentation by "merging" every odd row with its subsequent row:

$$
S \cong\left[\left(\begin{array}{c}
f_{i_{0}}^{\prime \prime}+g_{j_{0}}^{\prime} \\
0 \\
\vdots \\
0 \\
0
\end{array}\right), \quad\left(\begin{array}{c}
g_{j_{0}}^{\prime \prime} \\
f_{i_{1}}^{\prime} \\
\vdots \\
0 \\
0
\end{array}\right),\left(\begin{array}{c}
0 \\
f_{i_{1}}^{\prime \prime}+g_{j_{1}}^{\prime} \\
\vdots \\
0 \\
0
\end{array}\right), \ldots, \quad\left(\begin{array}{c}
0 \\
0 \\
\vdots \\
g_{j_{n-1}}^{\prime \prime} \\
f_{i_{n}}^{\prime}
\end{array}\right), \quad\left(\begin{array}{c}
0 \\
0 \\
\vdots \\
0 \\
f_{i_{n}}^{\prime \prime}+g_{j_{n}}^{\prime}
\end{array}\right)\right]_{\mathrm{R}}
$$

The same can be done with the horizontal stripes of any band module $B$ in (3.6):

$$
B \cong\left[\left(\begin{array}{c}
\left(f_{i_{1}}^{\prime \prime}+g_{j_{1}}^{\prime}\right) I \\
0 \\
\vdots \\
0
\end{array}\right), \quad\left(\begin{array}{c}
g_{j_{1}}^{\prime \prime} I \\
f_{i_{2}}^{\prime} I \\
\vdots \\
0
\end{array}\right), \ldots, \quad\left(\begin{array}{c}
0 \\
0 \\
\vdots \\
\left(f_{i_{n}}^{\prime \prime}+g_{j_{n}}^{\prime}\right) I
\end{array}\right), \quad\left(\begin{array}{c}
f_{i_{1}}^{\prime} J \\
0 \\
\vdots \\
g_{j_{n}}^{\prime \prime} I
\end{array}\right)\right]_{\mathrm{R}}
$$

Theorem 3.16. For the ring $\mathrm{R}=\mathbb{k} \llbracket x, y, u, v \rrbracket /\left(x y, x v, y u, u v, u^{2}, v^{2}\right)$ the classification of the indecomposable objects of $\mathrm{CM}(\mathrm{R})$ is the following.

- The modules $B(\omega, m, \lambda)$ and $S(\omega)$ are indecomposable. Moreover, any indecomposable Cohen-Macaulay $\mathrm{R}$-module is isomorphic to some band or some string module.

- $B(\omega, m, \lambda) \nRightarrow S\left(\omega^{\prime}\right)$ for any choice of parameters $\omega, \omega^{\prime}, m$ and $\lambda$.

- $S(\omega) \cong S\left(\omega^{\prime}\right)$ if and only if $\omega^{\prime}=\omega$ or $\omega^{\prime}=\omega^{o}$, where $\omega^{o}$ is the opposite word.

- $B(\omega, m, \lambda) \cong B\left(\omega^{\prime}, m^{\prime}, \lambda^{\prime}\right)$ if and only if $m=m^{\prime}, \lambda=\lambda^{\prime}$ and $\omega^{\prime}$ is given by a (possibly trivial) cyclic shift on all letters of $\omega$.

Proof. According to Theorem 2.1 the classification problem of indecomposable objects of $\mathrm{CM}(\mathrm{R})$ is equivalent to the matrix problem over the bunch of chains $\mathfrak{B}$ from Definition 3.1. More precisely, we had a diagram of categories and functors

$$
\mathrm{CM}(\mathrm{R}) \underset{\mathbb{G}}{\stackrel{\mathbb{F}}{\sim}} \operatorname{Tri}(\mathrm{R}) \stackrel{\mathbb{P}}{\longrightarrow} \operatorname{Rep}(\mathfrak{B})
$$

where $\mathbb{F}$ and $\mathbb{G}$ are mutually inverse equivalences of categories and $\mathbb{P}$ is a full functor preserving isomorphy classes and indecomposability of objects.

1. Indecomposable objects of the category $\operatorname{Rep}(\mathfrak{B})$ are classified by string and band data according to Theorem 3.4. Moreover, the indecomposable objects of $\operatorname{Rep}(\mathfrak{B})$ lying in the essential image of $\mathbb{P}$ are described by Remark 3.5 .

2. Let $w$ be the word of string or band datum in $\mathfrak{B}$, see Definition 3.2 and Definition 3.3. Note that $w$ is uniquely determined by the symbols in $\mathfrak{E}$ it contains. It follows that we may delete all subwords of the form $\gamma \sim \delta$ or $\delta \sim \gamma$ and relations - without loss of information. Now we can translate the remaining subwords as follows:

\begin{tabular}{|c|c|c|c|c|c|c|c|}
\hline$\xi_{0}$ & $\alpha_{i} \sim \xi_{i}$ & $\xi_{i} \sim \alpha_{i}$ & $\alpha_{\infty}$ & $\zeta_{0}$ & $\beta_{j} \sim \zeta_{j}$ & $\zeta_{j} \sim \beta_{j}$ & $\beta_{\infty}$ \\
\hline $\mathbf{x}_{0}$ & $\mathbf{x}_{i}^{+}$ & $\mathbf{x}_{i}^{-}$ & $\mathbf{x}_{\infty}$ & $\mathbf{y}_{0}$ & $\mathbf{y}_{j}^{+}$ & $\mathbf{y}_{j}^{-}$ & $\mathbf{y}_{\infty}$ \\
\hline
\end{tabular}

This table allows to pass from a word $w$ to a word $\omega$ as in Definitions 3.12 and 3.13 .

3. Consider a string datum $(w)$ (obeying the constraint from Remark 3.5) or a band datum $(w, m, \lambda)$. In Subsection 3.1 we explained the construction of the corresponding 
indecomposable object $\Theta=\left(\Theta_{x}, \Theta_{y}\right)$ of $\operatorname{Rep}(\mathfrak{B})$. Now we give the construction of a triple $\mathrm{T}=(N, V, \Theta)$ in $\operatorname{Tri}(\mathrm{R})$ such that $\mathbb{P}(\mathrm{T})=\Theta$. Let $m_{0}, m_{i}^{ \pm}, m_{\infty}, n_{0}, n_{j}^{ \pm}$respectively $n_{\infty}$ be the number of times the letter $\mathbf{x}_{0}, \mathbf{x}_{i}^{ \pm}, \mathbf{x}_{\infty}, \mathbf{y}_{0}, \mathbf{y}_{j}^{ \pm}$respectively $\mathbf{y}_{\infty}$ occurs in $w$. Let $t$ be the number of times $\gamma$ (or $\delta$ ) occurs in $w$. Then $\mathrm{T}=(N, V, \Theta)$, where

$$
\begin{aligned}
& \text { - } N=X_{0}^{m_{0}} \oplus \bigoplus_{i \in \mathbb{N}} X_{i}^{m_{i}^{+}+m_{i}^{-}} \oplus X_{\infty}^{m_{\infty}} \oplus Y_{0}^{n_{0}} \oplus \bigoplus_{j \in \mathbb{N}} Y_{j}^{n_{j}^{+}+n_{j}^{-}} \oplus Y_{\infty}^{n_{\infty}}, \\
& \text { - } V=\mathbb{k}^{t}, \\
& \text { - } \Theta=\left(\Theta_{x}, \Theta_{y}\right) \text {. }
\end{aligned}
$$

4. Now recall the construction of the indecomposable Cohen-Macaulay R-module $M=$ $\mathbb{G}(\mathrm{T})$. Consider a basis of a $\mathbb{k}$-vector space $N / \mathfrak{m} N$ given by the images of the distinguished generators of the indecomposable direct summands of $N$. Let $\pi: N \rightarrow N / \mathfrak{m} N$ be the canonical projection and $\widetilde{\Theta}:=\left(\begin{array}{c}\Theta_{x} \\ \Theta_{y}\end{array}\right): V \rightarrow N / \mathfrak{m} N$. By Theorem 1.19 we have:

$$
M:=\mathbb{G}(\mathrm{T})=\pi^{-1}(\operatorname{im}(\widetilde{\Theta})) \subseteq N .
$$

To compute $M$ we do the following:

(1) We multiply each entry of $\widetilde{\Theta}$ with its horizontal weight.

(2) For each subword $\epsilon^{\prime} \sim \epsilon^{\prime \prime}$ in $w$ such that $\epsilon^{\prime}, \epsilon^{\prime \prime} \in \mathfrak{E}$ we merge the two corresponding rows in $\widetilde{\Theta}$ and add their entries to each other.

(3) We translate all entries of the new matrix $\widetilde{\Theta}$ as follows:

\begin{tabular}{|c|c|c|c|c|c|c|c|}
\hline$\xi_{0}$ & $\xi_{k}$ & $\alpha_{\infty}$ & $\alpha_{k}$ & $\zeta_{0}$ & $\zeta_{k}$ & $\beta_{\infty}$ & $\beta_{k}$ \\
\hline$e_{1}$ & $x^{k}$ & $u$ & $u$ & $e_{2}$ & $y^{k}$ & $v$ & $v$ \\
\hline
\end{tabular}

Lemma 3.11 implies that $M$ is generated by the columns of the modified matrix $\widetilde{\Theta}$. Finally, observe that $M \cong(x+y) M$ in $\mathrm{R}-\bmod$. Thus, if $w$ is a string word containing one of the symbols $\xi_{0}$ or $\zeta_{0}$, one has to multiply the columns of $\widetilde{\Theta}$ with $(x+y)$ to obtain entries which lie in R. After permutation of rows in $\tilde{\Theta}$, one obtains exactly the presentations 3.6 . respectively (3.8) for the words in Definition 3.12 or 3.13 .

5. The statement about the isomorphy classes of string modules in $C M(R)$ is a direct translation of the corresponding result for the category $\operatorname{Rep}(\mathfrak{B})$ stated in Theorem 3.4 . Considering all pairwise non-equivalent band data $(w, m, \lambda)$, we may assume that the last letter of $w$ is $\gamma$ or $\delta$ by the equivalence conditions in Definition 3.3. Then Theorem 3.4 yields the isomorphism conditions for band modules $B(\omega, m, \lambda)$ as stated above.

6. Summing up, the key point of the proof of Theorem 3.16 is that we have the following isomorphisms in the category $\operatorname{Rep}(\mathfrak{B})$ by construction:

$$
\mathbb{P} \circ(\mathbb{F}(B(\omega, m, \lambda))) \cong\left(\Theta_{x}(w, m, \lambda), \Theta_{x}(w, m, \lambda)\right)
$$

for a band module $B(\omega, m, \lambda)$ from Definition 3.12 and

$$
\mathbb{P} \circ(\mathbb{F}(S(\omega))) \cong\left(\Theta_{x}(w), \Theta_{x}(w)\right) .
$$

for a string module $S(\omega)$ from Definition 3.13 .

Remark 3.17. By Lemma 3.11 any band module $B(\omega, m, \lambda)$ is locally free on the punctured spectrum. A string module $S(\omega)$ is not locally free on the punctured spectrum if and only if $\omega$ contains a letter $\mathbf{x}_{\infty}$ or $\mathbf{y}_{\infty}$. 
Example 3.18. In the following examples we translate the canonical forms of the preceding subsection into indecomposable Cohen-Macaulay modules over $\mathrm{R}$ as described in the proof of Theorem 3.16

(1) Let $(w, m, \lambda)$ be the band datum from Example 3.6 .

Then $w$ corresponds to $\omega=\mathbf{x}_{i}^{-} \mathbf{y}_{j_{1}}^{-} \mathbf{x}_{i}^{+} \mathbf{y}_{j_{2}}^{-}$and its band module is given by

$$
\begin{aligned}
B(\omega, m, \lambda) & \cong\left[\left(\begin{array}{c}
x^{i} I \\
0 \\
0 \\
v J
\end{array}\right),\left(\begin{array}{c}
u I \\
0 \\
y^{j_{1}} I \\
0
\end{array}\right),\left(\begin{array}{c}
0 \\
u I \\
v I \\
0
\end{array}\right),\left(\begin{array}{c}
0 \\
x^{i} I \\
0 \\
y^{j_{2}} I
\end{array}\right)\right]_{\mathrm{R}} \\
& \left.\cong\left[\begin{array}{c}
x^{i} I \\
v J
\end{array}\right),\left(\begin{array}{c}
\left(u+y^{j_{1}}\right) I \\
0
\end{array}\right),\left(\begin{array}{c}
v I \\
u I
\end{array}\right),\left(\begin{array}{c}
0 \\
\left(x^{i}+y^{j_{2}}\right) I
\end{array}\right)\right]_{\mathrm{R}}
\end{aligned}
$$

Here, $J$ denotes the Jordan block with eigenvalue $\lambda$ and $I$ the identity matrix, both of size $m$.

(2) Let $w$ be the string datum from Example 3.7.

Then $w$ corresponds to $\omega=\mathbf{x}_{i}^{-} \mathbf{y}_{j}^{-}$and its string module is given by

$$
S(\omega) \cong\left[\left(\begin{array}{c}
x^{i} \\
0
\end{array}\right),\left(\begin{array}{c}
u \\
y^{j}
\end{array}\right),\left(\begin{array}{l}
0 \\
v
\end{array}\right)\right]_{\mathrm{R}} \cong\left(x^{i}, u+y^{j}, v\right)_{\mathrm{R}}
$$

(3) Let $w$ be the string datum from Example 3.8.

Then $w$ corresponds to $\omega=\mathbf{x}_{0} \mathbf{y}_{j_{1}}^{-} \mathbf{x}_{i}^{-} \mathbf{y}_{j_{2}}^{+}$and its string module is given by

$$
\begin{aligned}
S(\omega) & \cong\left[\left(\begin{array}{c}
x \\
0 \\
y^{j_{1}+1} \\
0
\end{array}\right),\left(\begin{array}{c}
0 \\
x^{i+1} \\
v y \\
0
\end{array}\right),\left(\begin{array}{c}
0 \\
u x \\
0 \\
v y
\end{array}\right),\left(\begin{array}{c}
0 \\
0 \\
0 \\
y^{j_{2}+1}
\end{array}\right)\right]_{\mathrm{R}} \\
& \cong\left[\left(\begin{array}{c}
x+y^{j_{1}+1} \\
0
\end{array}\right),\left(\begin{array}{c}
v y \\
x^{i+1}
\end{array}\right),\left(\begin{array}{c}
0 \\
u x+v y
\end{array}\right),\left(\begin{array}{c}
0 \\
y^{j_{2}+1}
\end{array}\right)\right]_{\mathrm{R}}
\end{aligned}
$$

(4) Let $w$ be the string datum from Example 3.9 .

Then $w$ corresponds to $\omega=\mathbf{x}_{\infty} \mathbf{y}_{j}^{+} \mathbf{x}_{i}^{-} \mathbf{y}_{\infty}$ and its string module is given by

$$
S(\omega) \cong\left[\left(\begin{array}{c}
u \\
0 \\
v \\
0
\end{array}\right),\left(\begin{array}{c}
0 \\
x^{i} \\
y^{j} \\
0
\end{array}\right),\left(\begin{array}{l}
0 \\
u \\
0 \\
v
\end{array}\right)\right]_{\mathrm{R}} \cong\left[\left(\begin{array}{c}
u+v \\
0
\end{array}\right),\left(\begin{array}{c}
y^{j} \\
x^{i}
\end{array}\right),\left(\begin{array}{c}
0 \\
u+v
\end{array}\right)\right]_{\mathrm{R}}
$$

Remind that our original motivation was to describe indecomposable Cohen-Macaulay modules over the ring $\mathrm{P}=\mathbb{k} \llbracket x, y, z \rrbracket /\left(x y, z^{2}\right)$. Theorem 1.11, Lemma 3.11 and Theorem 3.16 yield a complete classification of indecomposable objects of $\mathrm{CM}(\mathrm{P})$.

Theorem 3.19. An indecomposable Cohen-Macaulay $\mathrm{P}$-module is either $\mathrm{P}$, or one of the band modules (3.6) respectively string modules (3.8). Moreover, in the formulae (3.6) and (3.8), the generation over $\mathrm{R}$ can be replaced by the generation over $\mathrm{P}$ (with the only exception of $\left.S\left(\mathbf{x}_{0} \mathbf{y}_{0}\right)\right)$. 
Remark 3.20. Any string or band module $M$ over $\mathrm{R}$ can be translated into a CohenMacaulay module $\mathbb{I}(M)$ over $\mathrm{P}$ as follows.

(1) Assume that $M=B(\omega, m, \lambda)$ or $M=S(\omega)$ where the string datum $\omega$ does not contain $\mathbf{x}_{0}$ or $\mathbf{y}_{0}$. We translate the entries of $M$ as follows:

\begin{tabular}{|c|c|c|c|}
\hline$x^{i}$ & $u$ & $y^{j}$ & $v$ \\
\hline$x^{i+1}$ & $x z$ & $y^{j+1}$ & $y z$ \\
\hline
\end{tabular}

There is a better presentation of $M$ if the following assumptions are satisfied:

- $\omega$ has even length and the sequence of signs $\tau_{k}, \sigma_{k}$ in $\omega$ alternates,

- if $\omega$ begins (respectively ends) with $\mathbf{x}_{\infty}$ or $\mathbf{y}_{\infty}$ then the first (respectively last) sign in $\omega$ is + (respectively - ).

In this case, we apply Remark 3.15 to $M$, replace $x z+y z$ by $z$, any entry of type $x^{i}$ by $x^{i-1}$ and any entry $y^{j}$ by $y^{j-1}$.

(2) If $M=S(\omega) \nRightarrow S\left(\mathbf{x}_{0} \mathbf{y}_{0}\right)$ is a string module such that the string datum $\omega$ contains $\mathbf{x}_{0}$ or $\mathbf{y}_{0}$, we translate all entries of $M$ as follows:

\begin{tabular}{|l|l|l|l|}
\hline$x^{i}$ & $u x$ & $y^{j}$ & $v y$ \\
\hline$x^{i}$ & $x z$ & $y^{j}$ & $y z$ \\
\hline
\end{tabular}

Remark 3.21. Theorem 3.19 remains valid for any curve singularity of type $\mathrm{P}_{2 r+1,2 s+1}$, where $r, s \in \mathbb{N}_{0} \cup\{\infty\}$, but string and band modules have to be redefined in the following way:

(1) The band and string modules over $\mathrm{P}_{2 r+1, \infty}$ are given by the Definitions 3.12 and 3.13 but their string and band words $\omega$ may only contain letters $\mathbf{x}_{i}$ such that $0 \leq i \leq r$ or $\mathbf{y}_{j}$, where $j \in \mathbb{N}_{0} \cup\{\infty\}$.

(2) Band and string data over $\mathrm{P}_{2 r+1,2 s+1}$, where $r, s \in \mathbb{N}_{0}$, may only contain the letters $\mathbf{x}_{i}$ such that $0 \leq i \leq r$ or $\mathbf{y}_{j}$ such that $0 \leq j \leq s$.

The method of this section can also be generalized using Bondarenko's work [6] to obtain an explicit classification of the indecomposable Cohen-Macaulay modules over the remaining curve singularities $\mathrm{P}_{2 r, q}$, where $r \in \mathbb{N}$ and $q \in \mathbb{N} \cup\{\infty\}$. This generalization is straightforward to carry out, but the explicit combinatorics are too space-consuming to be stated in the present article.

Example 3.22. In the following we apply Remark 3.20 to translate the string and band modules over $\mathrm{R}$ from 3.18 into indecomposable Cohen-Macaulay modules over $\mathrm{P}$.

(1) Let $(\omega, m, \lambda)$ be a band datum with $\omega=\mathbf{x}_{i}^{-} \mathbf{y}_{j_{1}}^{-} \mathbf{x}_{i}^{+} \mathbf{y}_{j_{2}}^{-}$. Then its band module $B=B(\omega, m, \lambda)$ translates over $\mathrm{P}$ into

$\mathbb{I}(B) \cong\left[\left(\begin{array}{c}x^{i+1} I \\ y z J\end{array}\right), \quad\left(\begin{array}{c}\left(x z+y^{j_{1}+1}\right) I \\ 0\end{array}\right), \quad\left(\begin{array}{c}x z I \\ y z I\end{array}\right), \quad\left(\begin{array}{c}0 \\ \left(x^{i+1}+y^{j_{2}+1}\right) I\end{array}\right)\right]_{\mathrm{P}}$

(2) Let $\omega=\mathbf{x}_{i}^{-} \mathbf{y}_{j}^{-}$. Then the string module $S=S(\omega)$ translates over P into

$$
\mathbb{I}(S) \cong\left(x^{i+1}, x z+y^{j+1}, y z\right)_{\mathrm{P}}
$$

(3) Let $\omega=\mathbf{x}_{0} \mathbf{y}_{j_{1}}^{-} \mathbf{x}_{i}^{-} \mathbf{y}_{j_{2}}^{+}$. Then the string module $S=S(\omega)$ translates over P into

$$
\mathbb{I}(S) \cong\left[\left(\begin{array}{c}
x+y^{j_{1}+1} \\
0
\end{array}\right), \quad\left(\begin{array}{c}
y z \\
x^{i+1}
\end{array}\right), \quad\left(\begin{array}{c}
0 \\
(x+y) z
\end{array}\right), \quad\left(\begin{array}{c}
0 \\
y^{j_{2}+1}
\end{array}\right)\right]_{\mathrm{P}}
$$


(4) Let $\omega=\mathbf{x}_{\infty} \mathbf{y}_{j}^{+} \mathbf{x}_{i}^{-} \mathbf{y}_{\infty}$. Then the string module $S=S(\omega)$ translates over $\mathrm{P}$ into

$$
\mathbb{I}(S) \cong\left[\left(\begin{array}{c}
x z \\
0 \\
y z \\
0
\end{array}\right), \quad\left(\begin{array}{c}
0 \\
x^{i} \\
y^{j} \\
0
\end{array}\right),\left(\begin{array}{c}
0 \\
x z \\
0 \\
y z
\end{array}\right)\right]_{\mathrm{P}} \cong\left[\left(\begin{array}{l}
z \\
0
\end{array}\right),\left(\begin{array}{l}
y^{j} \\
x^{i}
\end{array}\right),\left(\begin{array}{l}
0 \\
z
\end{array}\right)\right]_{\mathrm{P}}
$$

3.3. Induced Cohen-Macaulay modules of $\mathrm{T}_{\infty \infty}$ and their matrix factorizations. Our next motivation was to study Cohen-Macaulay modules over the hypersurface singularity $T=\mathbb{k} \llbracket a, b \rrbracket /\left(a^{2} b^{2}\right)$. At the beginning of Section 3 we have constructed a fully faithful functor $\mathbb{J}: \mathrm{CM}(\mathrm{R}) \longleftrightarrow \mathrm{CM}(\mathrm{T})$. Its explicit description, adapted to the combinatorics of bands and strings, was explained in Lemma 3.11, if $M=\left\langle w_{1}, \ldots, w_{t}\right\rangle_{\mathrm{R}} \subseteq \mathrm{S}^{k+l}$ then

$$
\mathbb{J}(M)=\left\langle w_{1}, \ldots, w_{t}, u w_{1}, \ldots, u w_{t}, v w_{1}, \ldots, v w_{t}\right\rangle_{\mathrm{T}} \subseteq \mathrm{S}^{k+l} .
$$

In fact, the number of generators of $\mathbb{J}(M)$ can be reduced.

Remark 3.23. Any string or band module $M$ over $\mathrm{R}$ can be translated into a CohenMacaulay module $\mathbb{J}(M)$ over $\mathrm{T}$ as follows.

- Let $M$ be a band module or a string module $S(\omega)$ such that $\omega$ does not $\mathbf{x}_{0}$ or $\mathbf{y}_{0}$. Then $\mathbb{J}(M)=\left\langle w_{1}, \ldots, w_{t}\right\rangle_{T}$. Moreover, we may replace all entries in every generator of $\mathbb{J}(M)$ by the table:

\begin{tabular}{|c|c|c|c|}
\hline$x^{i}$ & $u$ & $y^{j}$ & $v$ \\
\hline$a^{i+2}$ & $a^{2} b$ & $b^{j+2}$ & $a b^{2}$ \\
\hline
\end{tabular}

- Let $M=S(\omega)$ such that $\omega$ contains $\mathbf{x}_{0}$ or $\mathbf{y}_{0}$. For every column $w_{i}$ containing an entry $x$ respectively $y$, add $u w_{i}$ respectively $v w_{i}$ to the $\operatorname{span}\left\langle w_{1}, \ldots, w_{t}\right\rangle_{\mathrm{T}}$. Then $\mathbb{J}(M)$ is equal to this span with the additional generators. At last, we can translate all entries into elements of $\mathrm{T}$ using the table:

\begin{tabular}{|c|c|c|c|}
\hline$x^{i}$ & $u x$ & $y^{j}$ & $v y$ \\
\hline$a^{i+1}$ & $a^{2} b$ & $b^{j+1}$ & $a b^{2}$ \\
\hline
\end{tabular}

Example 3.24. Now we translate the string and band modules over R from 3.18 into indecomposable Cohen-Macaulay modules over T using Remark 3.23 .

(1) Let $(\omega, m, \lambda)$ be a band datum with $\omega=\mathbf{x}_{i}^{-} \mathbf{y}_{j_{1}}^{-} \mathbf{x}_{i}^{+} \mathbf{y}_{j_{2}}^{-}$. Then its band module $B=B(\omega, m, \lambda)$ translates over T into

$$
\mathbb{J}(B) \cong\left[\left(\begin{array}{c}
a^{i+2} I \\
a b^{2} J
\end{array}\right), \quad\left(\begin{array}{c}
\left(a^{2} b+b^{j_{1}+2}\right) I \\
0
\end{array}\right), \quad\left(\begin{array}{c}
a b^{2} I \\
a^{2} b I
\end{array}\right), \quad\left(\begin{array}{c}
0 \\
\left(a^{i+2}+b^{j_{2}+2}\right) I
\end{array}\right)\right]_{\mathrm{T}}
$$

(2) Let $\omega=\mathbf{x}_{i}^{-} \mathbf{y}_{j}^{-}$. Then the string module $S=S(\omega)$ translates over T into

$$
\mathbb{J}(S) \cong\left(a^{i+2}, a^{2} b+y^{j+2}, a b^{2}\right)_{\mathrm{T}}
$$

(3) Let $\omega=\mathbf{x}_{0} \mathbf{y}_{j_{1}}^{-} \mathbf{x}_{i}^{-} \mathbf{y}_{j_{2}}^{+}$. Then the string module $S=S(\omega)$ translates over $\mathrm{P}$ into

$$
\mathbb{J}(S) \cong\left[\left(\begin{array}{c}
a^{2}+b^{j_{1}+2} \\
0
\end{array}\right), \quad\left(\begin{array}{c}
a b^{2} \\
a^{i+2}
\end{array}\right), \quad\left(\begin{array}{c}
0 \\
a^{2} b+a b^{2}
\end{array}\right), \quad\left(\begin{array}{c}
0 \\
b^{j_{2}+2}
\end{array}\right), \quad\left(\begin{array}{c}
a^{2} b \\
0
\end{array}\right)\right]_{\mathrm{T}}
$$


(4) Let $\omega=\mathbf{x}_{\infty} \mathbf{y}_{j}^{+} \mathbf{x}_{i}^{-} \mathbf{y}_{\infty}$. Then the string module $S=S(\omega)$ translates over P into

$$
\begin{aligned}
\mathbb{J}(S) & \cong\left[\left(\begin{array}{c}
a^{2} b+a b^{2} \\
0
\end{array}\right), \quad\left(\begin{array}{l}
b^{j+2} \\
a^{i+2}
\end{array}\right),\left(\begin{array}{c}
0 \\
a^{2} b+a b^{2}
\end{array}\right)\right]_{\mathrm{T}} \\
& \cong\left[\left(\begin{array}{c}
a b \\
0
\end{array}\right), \quad\left(\begin{array}{c}
b^{j} \\
a^{i}
\end{array}\right),\left(\begin{array}{c}
0 \\
a b
\end{array}\right)\right]_{\mathrm{T}}
\end{aligned}
$$

Remark 3.25. There is an involution $\tau$ on $\mathrm{R}=\mathbb{k} \llbracket x, y, u, v \rrbracket /\left(x y, x v, y u, u v, u^{2}, v^{2}\right)$ which interchanges $x$ and $y, u$ and $v$. Restricted to $\mathrm{P}=\mathbb{k} \llbracket x, y, z \rrbracket /\left(x y, z^{2}\right) \subset \mathrm{R}, \tau$ is still an involution such that $\tau(z)=z$. The restriction of $\tau$ to $\mathrm{T}=\mathbb{k} \llbracket a, b \rrbracket /\left(a^{2} b^{2}\right) \subset A$ interchanges $a$ and $b$. Overall, $\tau$ induces an involution on the category of Cohen-Macaulay modules over R, P or T. The corresponding action of $\tau$ on words $\omega$ of string or band data of $\mathrm{CM}(\mathrm{R})$ is given by interchanging $\mathbf{x}$ and $\mathbf{y}$ in $\omega$.

In the following table, we list all indecomposable Cohen-Macaulay ideals of $\mathrm{R}$ and $\mathrm{P}$ (except $\mathrm{P}$ itself) and the corresponding ideals of $\mathrm{T}$ up to isomorphism and involution $\tau$. Let $i, j \in \mathbb{N}$ and $\lambda \in \mathbb{k}^{*}$. For all band data the multiplicity parameter $m$ is set to 1 .

\begin{tabular}{|l|l|l|l|}
\hline invariant of $\mathfrak{B}$ & ideal in R & ideal in P & ideal in T \\
\hline \hline $\mathbf{x}_{0}$ & $(x)$ & $(x)$ & $\left(a^{2}\right)$ \\
\hline $\mathbf{x}_{\infty}$ & $(u)$ & $(x z)$ & $\left(a^{2} b\right)$ \\
\hline $\mathbf{x}_{i}^{-}$ & $\left(x^{i}, u\right)$ & $\left(x^{i+1}, x z\right)$ & $\left(a^{i+2}, a^{2} b\right)$ \\
\hline $\mathbf{x}_{0} \mathbf{y}_{0}$ & $(1)$ & $(x+y, x z)$ & $\left(a^{2}+b^{2}, a^{2} b, a b^{2}\right)$ \\
\hline $\mathbf{x}_{i}^{+} \mathbf{y}_{0}$ & $\left(u x, x^{i+1}+y\right)$ & $\left(x z, x^{i+1}+y\right)$ & $\left(a^{2} b, a^{i+2}+b^{2}, a b^{2}\right)$ \\
\hline $\mathbf{x}_{i}^{+} \mathbf{y}_{j}^{-}$ & $\left(u, x^{i}+y^{j}, v\right)$ & $\left(x z, x^{i+1}+y^{j+1}, y z\right)$ & $\left(a^{2} b, a^{i+2}+b^{j+2}, a b^{2}\right)$ \\
\hline $\mathbf{x}_{i}^{-} \mathbf{y}_{0}$ & $\left(x^{i+1}, u x+y\right)$ & $\left(x^{i+1}, x z+y\right)$ & $i=1:\left(a^{3}, a^{2} b+b^{2}\right)$ \\
\hline $\mathbf{x}_{i}^{-} \mathbf{y}_{j}^{-}$ & $\left(x^{i}, u+y^{j}, v\right)$ & $\left(x^{i+1}, x z+y^{j+1}, y z\right)$ & $\left(a^{i+2}, a^{2} b+b^{j+2}, a b^{2}\right)$ \\
\hline $\mathbf{x}_{i}^{-} \mathbf{y}_{j}^{+}$ & $\left(x^{i}, u+v, y^{j}\right)$ & $\left(x^{i}, z, y^{j}\right)$ & $\left(a^{i+2}, a^{2} b+a b^{2}, b^{j+2}\right)$ \\
\hline $\mathbf{x}_{\infty} \mathbf{y}_{0}$ & $(u x+y)$ & $(x z+y)$ & $\left(a^{2} b+b^{2}, a b^{2}\right)$ \\
\hline $\mathbf{x}_{\infty} \mathbf{y}_{j}^{-}$ & $\left(u+y^{j}, v\right)$ & $\left(x z+y^{j+1}, y z\right)$ & $\left(a^{2} b+b^{j+2}, a b^{2}\right)$ \\
\hline $\mathbf{x}_{\infty} \mathbf{y}_{j}^{+}$ & $\left(u+v, y^{j}\right)$ & $\left(z, y^{j}\right)$ & $\left(a^{2} b+a b^{2}, b^{j+2}\right)$ \\
\hline $\mathbf{x}_{\infty} \mathbf{y}_{\infty}$ & $(u+v)$ & $(z)$ & $\left(a^{2} b+a b^{2}\right) \cong(a b)$ \\
\hline$\left(\mathbf{x}_{i}^{-} \mathbf{y}_{j}^{-}, 1, \lambda\right)$ & $\left(x^{i}+\lambda v, y^{j}+u\right)$ & $\left(x^{i+1}+\lambda y z, y^{j+1}+x z\right)$ & $\left(a^{i+2}+\lambda a b^{2}, b^{j+2}+a^{2} b\right)$ \\
\hline$\left(\mathbf{x}_{i}^{-} \mathbf{y}_{j}^{+}, 1, \lambda\right)$ & $\left(x^{i}+\lambda y^{j}, u+v\right)$ & $\left(x^{i}+\lambda y^{j}, z\right)$ & $\left(a^{i+2}+\lambda b^{j+2}, a^{2} b+a b^{2}\right)$ \\
\hline
\end{tabular}

Remark 3.26. The above list does not contain all indecomposable ideals of $T$. For example, the ideal

$$
\left(a^{2}+\lambda b^{2}, a^{2} b+a b^{2}\right)
$$

is not induced by an ideal in $\mathrm{R}$ for any $\lambda \in \mathbb{k}^{*}$. 
Let $\underline{\mathrm{MF}}\left(a^{2} b^{2}\right)$ be the homotopy category of matrix factorizations of $a^{2} b^{2}$. By a result of Eisenbud [18] there is an equivalence of triangulated categories $\underline{\mathrm{CM}}(\mathrm{T}) \stackrel{\sim}{\longrightarrow} \mathrm{MF}\left(a^{2} b^{2}\right)$. In the following table, we list the matrix factorizations of $a^{2} b^{2}$ which originate from an indecomposable ideal in $\mathrm{R}$ (up to isomorphism and involution). Let $i, j \in \mathbb{N}$ and $\lambda \in \mathbb{k}^{*}$.

\begin{tabular}{|c|c|}
\hline ideal in $\mathbb{k} \llbracket a, b \rrbracket /\left(a^{2} b^{2}\right)$ & matrix factorization $(\phi, \psi)$ of $a^{2} b^{2}$ \\
\hline$\left(a^{2}\right)$ & $\left(b^{2}\right)\left(a^{2}\right)$ \\
\hline$\left(a^{2} b\right)$ & $(b)\left(a^{2} b\right)$ \\
\hline$\left(a^{i+2}, a^{2} b\right)$ & $\left(\begin{array}{cc}b & 0 \\
-a^{i} & b\end{array}\right)\left(\begin{array}{cc}a^{2} b & 0 \\
a^{i+2} & a^{2} b\end{array}\right)$ \\
\hline$\left(a^{i+1}+b^{j+1}, a^{2} b, a b^{2}\right)$ & $\left(\begin{array}{ccc}a b & 0 & 0 \\
-a^{i} & b & 0 \\
-b^{j} & 0 & a\end{array}\right)\left(\begin{array}{ccc}a b & 0 & 0 \\
a^{i+1} & a^{2} b & 0 \\
b^{j+1} & 0 & a b^{2}\end{array}\right)$ \\
\hline$\left(a^{i+3}, a^{2} b+b^{2}, a b^{2}\right)$ & $\left(\begin{array}{ccc}b & 0 & 0 \\
-a^{i+1} & a b & 0 \\
0 & -b & a\end{array}\right)\left(\begin{array}{ccc}a^{2} b & 0 & 0 \\
a^{i+2} & a b & 0 \\
a^{i+1} b & b^{2} & a b^{2}\end{array}\right)$ \\
\hline$\left(a^{3}, a^{2} b+b^{2}\right)$ & $\left(\begin{array}{cc}a b & 0 \\
-a^{2} & a^{2} b\end{array}\right)\left(\begin{array}{cc}a b & 0 \\
-a & b\end{array}\right)$ \\
\hline$\left(a^{i+2}, a^{2} b+b^{j+2}, a b^{2}\right)$ & $\left(\begin{array}{ccc}b & 0 & 0 \\
-a^{i} & a b & 0 \\
-a^{i-1} b^{j} & -b^{j+1} & a\end{array}\right)\left(\begin{array}{ccc}a^{2} b & 0 & 0 \\
a^{i+1} & a b & 0 \\
0 & b^{j+2} & a b^{2}\end{array}\right)$ \\
\hline$\left(a^{i+2}, b^{j+2}, a^{2} b+a b^{2}\right)$ & $\left(\begin{array}{ccc}b & 0 & 0 \\
0 & a & 0 \\
-a^{i} & -b^{j} & a b\end{array}\right)\left(\begin{array}{ccc}a^{2} b & 0 & 0 \\
0 & a b^{2} & 0 \\
a^{i+1} & b^{j+1} & a b\end{array}\right)$ \\
\hline$\left(a^{2} b+b^{j+1}, a b^{2}\right)$ & $\left(\begin{array}{cc}a b & 0 \\
-b^{j} & a\end{array}\right)\left(\begin{array}{cc}a b & 0 \\
b^{j+1} & a b^{2}\end{array}\right)$ \\
\hline$\left(b^{j+2}, a^{2} b+a b^{2}\right)$ & $\left(\begin{array}{cc}a & 0 \\
-b^{j} & a b\end{array}\right)\left(\begin{array}{cc}a b^{2} & 0 \\
b^{j+1} & a b\end{array}\right)$ \\
\hline$(a b)$ & $(a b)(a b)$ \\
\hline$\left(a^{i+2}+\lambda a b^{2}, a^{2} b+b^{j+2}\right)$ & $\left(\begin{array}{cc}a b & -b^{j+1} \\
-a^{i+1} & \lambda a b\end{array}\right)\left(\begin{array}{cc}a b & \lambda^{-1} b^{j+1} \\
\lambda^{-1} a^{i+1} & \lambda^{-1} a b\end{array}\right)$ \\
\hline$\left(a^{i+2}+\lambda b^{j+2}, a^{2} b+a b^{2}\right)$ & $\left(\begin{array}{cc}a b & 0 \\
-\lambda b^{j+1}-a^{i+1} & a b\end{array}\right)\left(\begin{array}{cc}a b & 0 \\
\lambda b^{j+1}+a^{i+1} & a b\end{array}\right)$ \\
\hline
\end{tabular}


Remark 3.27. By Knörrer's periodicity [24] the functor

$$
\begin{aligned}
& \underline{\mathrm{MF}}\left(a^{2} b^{2}\right) \stackrel{\sim}{\longrightarrow} \underline{\mathrm{MF}}\left(a^{2} b^{2}+u v\right) \\
& (\phi, \psi) \longmapsto\left(\begin{array}{cc}
\phi & -u \cdot I \\
v \cdot I & \psi
\end{array}\right)\left(\begin{array}{cc}
\psi & v \cdot I \\
-u \cdot I & \phi
\end{array}\right)
\end{aligned}
$$

is an equivalence of triangulated categories. It allows to get explicit families of matrix factorizations of any potential of type

$$
a^{2} b^{2}+u_{1} v_{1}+\ldots+u_{d} v_{d} \in \mathbb{k} \llbracket a, b, u_{1}, \ldots, u_{d}, v_{1}, \ldots, v_{d} \rrbracket .
$$

Remark 3.28. Let $\operatorname{char}(\mathbb{k}) \neq 2$. Then there is a ring isomorphism

$$
\mathbb{k} \llbracket a, b, c \rrbracket /\left(a^{2} b^{2}-c^{2}\right) \cong \mathbb{k} \llbracket x, y, z \rrbracket /\left(z^{2}-x y z\right)=: \mathrm{T}_{\infty \infty 2} .
$$

The indecomposable Cohen-Macaulay modules over the surface singularity $\mathrm{T}_{\infty \infty 2}$ have been classified in [11. On the other hand, Knörrer's correspondence [24] relates $\mathrm{T}_{\infty \infty 2}$ to $\mathrm{T}_{\infty \infty}$ by a restriction functor

$$
\underline{\mathrm{MF}}\left(a^{2} b^{2}-c^{2}\right) \longrightarrow \underline{\mathrm{MF}}\left(a^{2} b^{2}\right),
$$

such that every indecomposable matrix factorization of $a^{2} b^{2}$ appears as a direct summand of the restriction of some indecomposable matrix factorization of $a^{2} b^{2}-c^{2}$. With some efforts, one can compute the matrix factorizations of $a^{2} b^{2}$ corresponding to CohenMacaulay $\mathrm{T}_{\infty \infty 2^{-}}$-modules of small rank. However, it is not straightforward to derive all indecomposable matrix factorizations of $a^{2} b^{2}$ by this approach.

Remark 3.29. The approach to classify indecomposable Cohen-Macaulay modules using the technique of tame matrix problems is close in spirit to the study of torsion free sheaves on degenerations of elliptic curves. See [5] for a survey of the corresponding results and methods.

3.4. Some remarks on the stable category of Cohen-Macaulay modules. Let $(A, \mathfrak{m})$ be a Gorenstein singularity (of any Krull dimension $d$ ). By a result of Buchweitz [8], the natural functor

$$
\underline{\mathrm{CM}}(A) \longrightarrow D_{s g}(A):=\frac{D^{b}(\bmod (A))}{\operatorname{Perf}(A)}
$$

is an equivalence of triangulated categories. If the singularity $A$ is not isolated, then $\mathrm{CM}(A)$ is Hom-infinite [1]. On the other hand, the stable category of Cohen-Macaulay modules $\mathrm{CM}^{\text {lf }}(A)$ is always a Hom-finite triangulated subcategory of $\underline{\mathrm{CM}}(A)$. By a result of Auslander [1], the category $\underline{\mathrm{CM}}^{\mathrm{lf}}(A)$ is $(d-1)$-Calabi-Yau. This means that for any objects $M_{1}$ and $M_{2}$ of $\mathrm{CM}^{\text {lf }}(A)$ we have an isomorphism

$$
\underline{\operatorname{Hom}}_{A}\left(M_{1}, M_{2}\right) \cong \mathbb{D}\left(\underline{\operatorname{Hom}}_{A}\left(M_{2}, \Sigma^{d-1}\left(M_{1}\right)\right)\right),
$$

functorial in both arguments $M_{1}$ and $M_{2}$, where $\mathbb{D}$ is the Matlis duality functor and $\Sigma=\Omega^{-1}$ is the suspension functor. In particular, if $A$ is a Gorenstein curve singularity, then for any $M \in \underline{\mathrm{CM}}^{\mathrm{If}}(A)$ the algebra $\underline{\operatorname{End}}_{A}(M)$ is Frobenius. Thus, Theorem 2.1 gives a family of examples of representation tame 0-Calabi-Yau triangulated categories and Theorem 3.19 provides a complete and explicit description of indecomposable objects in one of such categories $\underline{\mathrm{CM}}^{\mathrm{lf}}(\mathrm{P})$ for $\mathrm{P}=\mathbb{k} \llbracket x, y, z \rrbracket /\left(x y, z^{2}\right)$. 


\section{REFERENCES}

[1] M. Auslander, Functors and morphisms determined by objects, In Representation Theory of Algebras, Lecture Notes in Pure Appl. Math. 37, (1978), 1-244.

[2] M. Auslander, Isolated singularities and existence of almost split sequences, In Representation Theory II, Proc. ICRA IV, Springer Lecture Notes in Math. 1178 (1986), 194-242.

[3] H. Bass, On the ubiquity of Gorenstein rings, Math. Z. 82 (1963) 8-28.

[4] N. Bourbaki, Commutative Algebra. Chapters 17, Elements of Mathematics, Springer-Verlag, Berlin, 1989.

[5] L. Bodnarchuk, I. Burban, Yu. Drozd and G.-M. Greuel, Vector bundles and torsion free sheaves on degenerations of elliptic curves, Global aspects of complex geometry, 83-128, Springer, Berlin, 2006.

[6] V. M. Bondarenko, Representations of bundles of semichained sets and their applications, Algebra $\mathrm{i}$ Analiz 3 (1991), no. 5, 38-61.

[7] W. Bruns and J. Herzog, Cohen-Macaulay Rings, Cambridge Studies in Advanced Mathematics 39, Cambridge Univ. Press, 1993.

[8] R.-O. Buchweitz, Maximal Cohen-Macaulay modules and Tate-Cohomology over Gorenstein rings, Preprint 1987.

[9] R. Buchweitz, G.-M. Greuel and F.-O. Schreyer, Cohen-Macaulay modules on hypersurface singularities II, Invent. Math. 88 (1987), no. 1, 165-182.

[10] I. Burban and Yu. Drozd, Maximal Cohen-Macaulay modules over surface singularities, Trends in representation theory of algebras and related topics, 101-166, EMS Ser. Congr. Rep., Eur. Math. Soc., Zürich (2008).

[11] I. Burban and Yu. Drozd, Maximal Cohen-Macaulay modules over non-isolated surface singularities, arXiv:1002.3042.

[12] I. Burban, O. Iyama, B. Keller and I. Reiten, Cluster tilting for one-dimensional hypersurface singularities, Adv. Math. 217 (2008), no. 6, 2443-2484.

[13] Yu. Drozd, Matrix problems and categories of matrices, Investigations on the theory of representations, Zap. Nauchn. Semin. Leningr. Otd. Mat. Inst. Steklova 28, 144-153 (1972).

[14] Yu. Drozd and G.-M. Greuel, Tame-wild dichotomy for Cohen-Macaulay modules, Math. Ann. 294 (1992), no. 3, 387-394.

[15] Yu. Drozd and G.-M. Greuel, Cohen-Macaulay module type, Compositio Math. 89 (1993), no. 3, 315-338.

[16] Yu. Drozd, G.-M. Greuel and I. Kashuba, On Cohen-Macaulay modules on surface singularities, Mosc. Math. J. 3 (2003), no. 2, 397-418.

[17] Yu. Drozd and A. Roiter, Commutative rings with a finite number of indecomposable integral representations, Izv. Akad. Nauk SSSR Ser. Mat. 31 (1967), 783-798.

[18] D. Eisenbud, Homological algebra on a complete intersection, with an application to group representations, Trans. Amer. Math. Soc. 260 (1980), no. 1, 35-64.

[19] G.-M. Greuel and H. Knörrer, Einfache Kurvensingularitäten und torsionsfreie Moduln, Math. Ann. 270 (1985), no. 3, 417-425.

[20] G.-M. Greuel and H. Kröning, Simple singularities in positive characteristic, Math. Z. 203 (1990), no. 2, 339-354.

[21] H. Jacobinski, Sur les ordres commutatifs avec un nombre fini de réseaux indécomposables, Acta Math. 118 (1967), 1-31.

[22] C. Kahn, Reflexive modules on minimally elliptic singularities, Math. Ann. 285 (1989), no. 1, 141-160.

[23] K. Kiyek and G. Steinke, Einfache Kurvensingularitäten in beliebiger Charakteristik, Arch. Math. 45 (1985), no. 6, 565-573.

[24] H. Knörrer, Cohen-Macaulay modules on hypersurface singularities I, Invent. Math 88 (1987), no. 1, 153-164.

[25] G. Leuschke and R. Wiegand, Cohen-Macaulay representations, AMS Mathematical Surveys and Monographs 181 (2012).

[26] G. Leuschke and R. Wiegand, Local rings of bounded Cohen-Macaulay type, Algebr. Represent. Theory 8 (2005), no. 2, 225-238.

[27] Y. Yoshino, Cohen-Macaulay Modules over Cohen-Macaulay Rings, London Mathematical Society Lecture Note Series 146, Cambridge Univ. Press, 1990. 
Universität Zu Köln, Mathematisches Institut, Weyertal 86-90, D-50931 Köln, Germany

E-mail address: burban@math.uni-koeln.de

Universität zU Köln, Mathematisches Institut, Weyertal 86-90, D-50931 Köln, Germany

E-mail address: wgnedin@math.uni-koeln.de 